\title{
Large-eddy simulation of a curved open-channel flow over topography
}

\author{
W. van Balen, ${ }^{1, a)}$ W. S. J. Uijttewaal, ${ }^{1}$ and K. Blanckaert ${ }^{1,2}$ \\ ${ }^{1}$ Faculty of Civil Engineering and Geosciences, Delft University of Technology, Delft 2600, The Netherlands \\ ${ }^{2}$ ICARE-ENAC, Ecole Polytechnique Fédérale, CH-1015 Lausanne, Switzerland
}

(Received 29 October 2009; accepted 6 May 2010; published online 23 July 2010)

\begin{abstract}
Large-eddy simulation (LES) is performed of a curved open-channel flow over topography based on the laboratory experiment by Blanckaert ["Topographic steering, flow circulation, velocity redistribution and bed topography in sharp meander bends," Water Resour. Res., doi:10.1029/ 2009WR008303 (in press)]. In the experiment, the large-scale bed topography had developed to a more or less stationary shape which was prescribed in the LES model as boundary conditions neglecting the small-scale dune forms by means of a straightforward immersed boundary scheme in combination with a simple wall-modeling approach. The small-scale dunes are accounted for in the numerical model by means of parametrization. Sensitivity of the flow to this roughness parametrization is examined by simulating the flow for three different roughness heights. It was found that, notwithstanding the coarse method of representing the dune forms, the qualitative agreement of the experimental results and the LES results is rather good. Comparison of the LES results with the Reynolds averaged numerical simulation results of Zeng et al. ["Flow and bathymetry in sharp open-channel bends: Experiments and predictions," Water Resour. Res. 44, W09401, doi:10.1029/2007WR006303 (2008)] reveals surprisingly good agreement. This good agreement is explained by the minor importance of turbulence stress gradients in the contribution to the transverse and streamwise momentum balance. Moreover, it is found that in the bend the structure of the Reynolds stress tensor shows a tendency toward isotropy which enhances the performance of isotropic eddy viscosity closure models of turbulence. This observation is remarkable since highly anisotropic turbulence might well be expected considering the complex nature of the geometry. Furthermore, the LES results reveal a pronounced recirculation zone near the convex inner bank of the flume due to the shallowness of the flow and strong curvature of the flume. At the interface between the recirculation zone and the main flow, a curved mixing layer is identified as well as strong upwelling flow motion that is accompanied with large production of turbulent kinetic energy. (C) 2010 American Institute of Physics. [doi:10.1063/1.3459152]
\end{abstract}

\section{INTRODUCTION}

A natural river flow can generally be characterized as an open-channel flow at a very high Reynolds number having a large width-to-depth ratio and being strongly curved locally. In addition, the river bed often has a very complex shape and strongly deforms as the river currents flow over it. This interaction between the river flow and the river bed has smallscale consequences as well as large-scale consequences. On a small scale, the near-bed turbulence behavior of the flow gives rise to the existence of so-called ripples or dunes. On a large scale, the erosion and sedimentation pattern of the bed is strongly influenced by the secondary flow that is induced by centripetal forces in curved parts of the river. As a result, the topography of the bed consists of a shallow area near the inner bank of a bend (a so-called pointbar) and a deep area near the outer bank of a bend (a so-called pool).

In order to acquire knowledge of this very complex flow type, river flow is often downscaled to laboratory dimensions to enable the investigation of the several aspects of the flow properties under optimized and controlled conditions. Ample results of experimental studies on curved open-channel

\footnotetext{
${ }^{a)}$ Electronic mail: wimvanbalentu@gmail.com. Present address: $\mathrm{HKV}_{\text {Consultants, Lelystad, The Netherlands. }}$
}

flows, either through a rectangular flume or over topography, are published in literature. Among others, the work of Rozovskii, ${ }^{1}$ Odgaard and Bergs, ${ }^{2}$ Blanckaert and Graf, ${ }^{3}$ Booij, ${ }^{4}$ and Abad and Garcia, ${ }^{5,6}$ can be mentioned in this respect. These studies have comprehensively contributed to the understanding of the mean flow and turbulence features in curved open channels, but are often based on rather inaccurate velocity measurements on coarse measuring grids.

The rapid increase in computational potential during the past few decades allows for the use of large-eddy simulation (LES) of these complex high Reynolds number flows. Although LESs have become rather common for the simulation of straight open-channel flows (cf. Refs. 7-9 and many others), it is yet rarely used for the simulation of curved openchannel flows. To the knowledge of the authors, only the simulation of meandering open-channel flow by Stoesser et al. ${ }^{10}$ and the simulation of single-bend open-channel flow by van Balen et al. ${ }^{11}$ can be mentioned. LES though is a very useful tool to study these types of flow, particularly since results from Reynolds averaged numerical simulations (RANSs) are not always very satisfactory (cf. Refs. 4 and 12). Moreover, LES can complement the experimental findings which are often restricted to several cross-sections or even a few profiles. 
The present paper focuses on the large-eddy simulation of an experiment of a strongly curved open-channel flow over topography of which the results have recently been reported by Blanckaert. ${ }^{13}$ The experiment was carried out in a laboratory flume with a movable sand bed and vertical sidewalls. The movable bed exhibited both the small-scale dunes on the bed and the large-scale pointbar-pool structure of the bed along the bend. Since the used LES model is not yet able to take directly into account the time-dependent dynamic behavior of the dunes on the bed, these are just modeled using a simple parametrization in terms of roughness height. The time-averaged bottom topography is prescribed in the LES computation as a boundary embedded in the computational grid. A first simulation of this experiment has yet been performed by Zeng et al. ${ }^{14}$ using a RANS model with a oneequation turbulence closure model in an attempt to mimic the evolution of the bed. Although Blanckaert ${ }^{13}$ measured the three-dimensional flow structure, the analysis reported in Ref. 13 mainly focused on the depth-averaged statistics of the flow for the purpose of linking the experimental results to two-dimensional engineering numerical models.

The aim of the present paper is to contribute to the existing body of literature on curved open-channel flows in general and the papers by Blanckaert ${ }^{13}$ and Zeng et al. ${ }^{14}$ in particular by answering a fourfold research question. First, what is the three-dimensional flow structure of a strongly curved open-channel flow over topography? Second, what are the differences in performance between a LES approach and a RANS approach? Third, what are the dominant mechanisms with respect to the transport of momentum? And fourth, what is the structure of the turbulent motion as regards its kinetic energy, its anisotropy, and the boundary shear stresses?

These four research questions are subsumed in separate sections in the paper. After a description of the experimental setup and the large-eddy simulation model in Sec. II, the flow phenomenology is depicted in Sec. III In Secs. IV and $\mathrm{V}$, the mechanisms of the transport of momentum and the structure of the turbulence are analyzed, respectively. The obtained results are summarized in Sec. VI

\section{MODEL DESCRIPTION}

\section{A. Physical domain}

The laboratory flume used by Blanckaert ${ }^{13}$ consists of a straight inflow section of $9 \mathrm{~m}$, a curved section of $193^{\circ}$, and a straight outflow section of $5 \mathrm{~m}$. In the numerical model, the inflow section and outflow section are shortened to a length of $3.8 \mathrm{~m}$ each to save computational costs (see Fig. 1). The bed and the water surface topography were measured using acoustic limnimeters. However, despite the dense measuring grid, the spatial resolution was not sufficient to resolve the details of the dunes superposed on the macroscale topography. Therefore, the small-scale dunes will be parametrized. The vertical sidewalls of the flume are hydraulically smooth. The sand particles that are used have a diameter ranging from 1.6-to $2.2 \mathrm{~mm}$ with an average value of about $2.0 \mathrm{~mm}$. The mean water depth $H$ is $0.141 \mathrm{~m}$ and the discharge $Q$ is $89 \mathrm{l} / \mathrm{s}$. Given the width of the flume $B=1.3 \mathrm{~m}$, a bulk

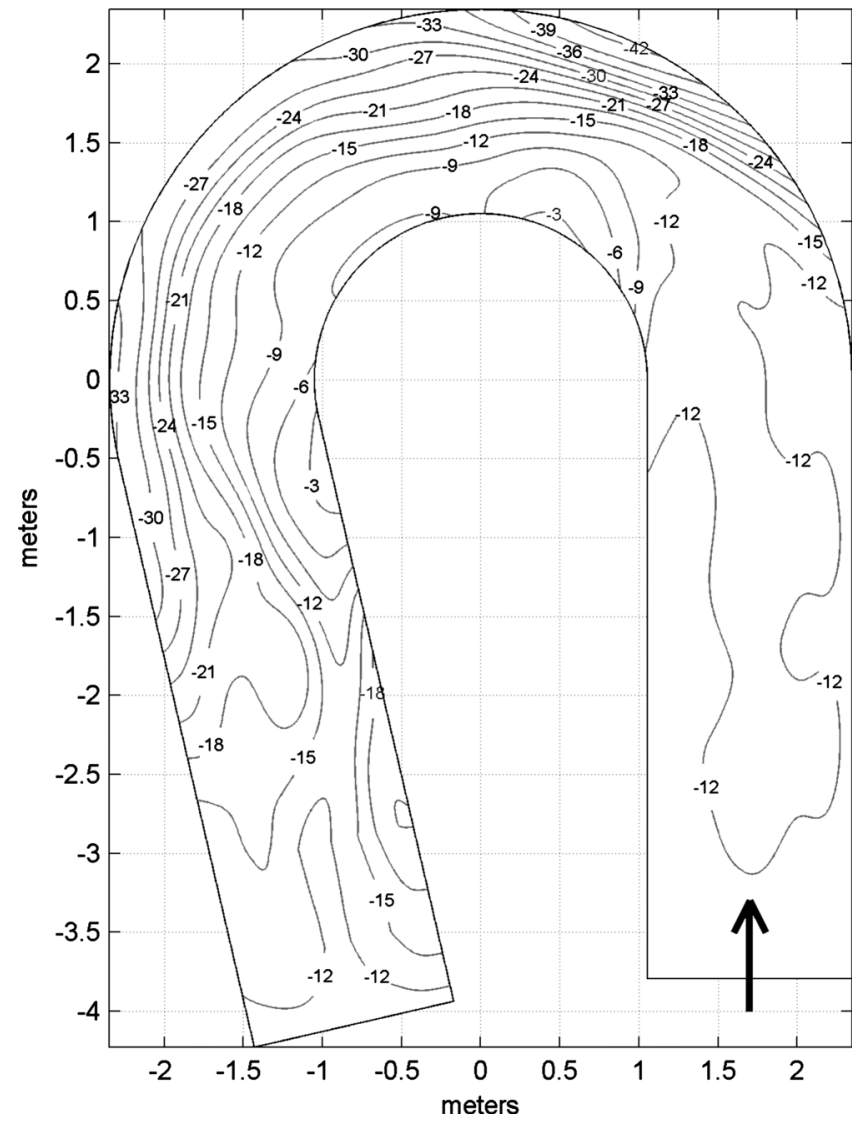

FIG. 1. Bottom topography given as the height (in centimeter) of the bottom using the water level as a reference level. The arrow denotes the flow direction.

velocity $V_{\mathrm{av}}$ can be calculated as $V_{\mathrm{av}}=Q / B H=0.49 \mathrm{~m} / \mathrm{s}$. The Reynolds number based on this velocity and the mean water depth is 68000 .

\section{B. Numerical model}

A cylindrical reference system with $r, \theta$, and $z$ as the transverse, streamwise, and vertical directions is adopted (Fig. 2). The associated velocity vector has the components $u, v$, and $w$ as the respective transverse, streamwise, and vertical velocity. For the LES model, the Navier-Stokes equations, formulated in cylindrical coordinates and filtered by a top-hat filter with the grid spacing as filter length, are solved. In straight parts of the geometry, we let $1 / R \rightarrow 0$ such that the coordinate system changes to a Cartesian one with coordinates $x, y$, and $z$ as the respective transverse, streamwise, and vertical directions (see Fig. 2, right panel). Notice that $1 / R \rightarrow 0$ yields $r \partial \theta \rightarrow \partial y$ for the equations of motion (cf. Ref. 11).

The used LES model is a finite volume model (Refs. 15 and 16). The incompressible Navier-Stokes equations are numerically integrated using the midpoint rule on a structured, regular, staggered grid within the context of the pressure-correction algorithm. As a matter of fact, this procedure results in the spatial discretization of the domain following the second order central scheme. The equations are integrated in time using the explicit second order AdamsBashforth scheme. More details on the equations and their 

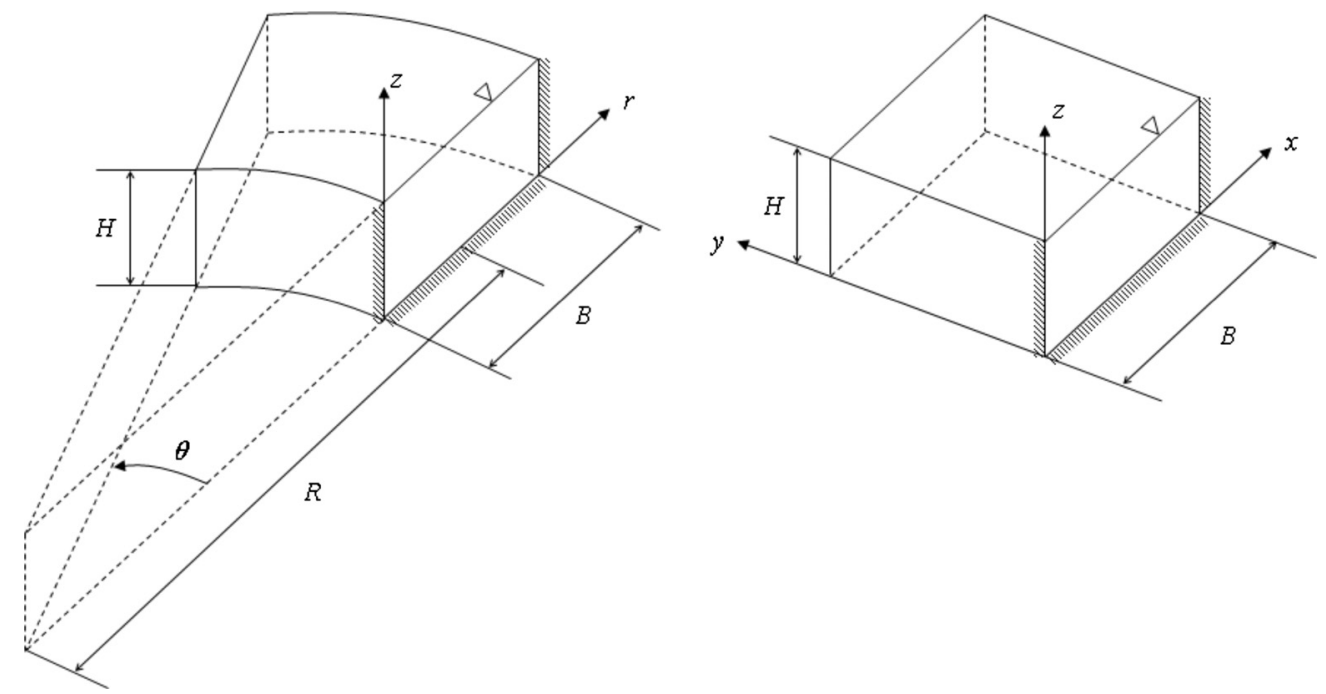

FIG. 2. Left panel: cylindrical coordinates system with $R$ the radius at the channel axis. Right panel: Cartesian coordinates system. $B$ is the width and $H$ is the height of the channel.

discretization can be found in Ref. 15. The standard Smagorinsky model is used for the modeling of the subgrid stresses. The associated Smagorinsky constant is chosen equal to the standard value of 0.1 .

The choice for the standard Smagorinsky model is mainly based on the findings of Ref. 12, who have conducted a numerical study of flow in the same laboratory infrastructure, be it through a flume with a rectangular cross-section instead of an irregular cross-section. Their simulations, obtained with LES with either the standard Smagorinsky model or the dynamic Smagorinsky model, indicate that the results are almost independent of the choice for the Smagorinsky model. This observation is also in line with the findings of Ref. 11, who have investigated a LES study on mildly curved open-channel flow using two different subgrid models. Recall that for the standard Smagorinsky model only turbulence isotropy on the subgrid scale is assumed; it still facilitates possible large-scale turbulence anisotropy. The big advantage of the standard Smagorinsky model is that is computationally inexpensive.

\section{Boundary conditions}

The free surface is treated as a horizontal, impermeable rigid lid where free-slip conditions are applied. The use of such a rigid lid is justified as long as the gradients of the water surface are small enough. This approach is often used for straight open-channel flows (cf. Refs. 7, 8, and 17 and many others) as well as for curved open-channel flows (cf. Refs. 10 and 18). Nonetheless, it should be noted that there is a continuity error accompanying this treatment of the free surface. However, Demuren and Rodi ${ }^{18}$ argued that as long as the superelevation of the free surface is less than $10 \%$ of the channel depth (which is the case in the current experiment), this error is small.

In order to minimize this continuity error, a fictitious bed topography was implemented that was obtained by subtracting enzovoort. Since the gradients in water surface elevation are small compared to those in the bed topography, this only marginally affects the topographic steering of the flow by gradients in the bed topography. The gradients in the water surface were small though. The bed topography as used in the numerical model is shown in Fig. 1. In this picture, the values denote the height (in centimeter) of the bottom using the water level as the reference nil level.

Solid walls are treated with the wall-function approach instead of refining the grid to resolve the near-wall flow structures up to the viscous sublayer. The latter approach would require a finer local mesh which would result in higher computational costs. For the hydraulically smooth vertical sidewalls, the standard law-of-the-wall is used with the viscous sublayer, the buffer layer, and the logarithmic layer incorporated following

$$
\begin{aligned}
& v_{n}^{+}=z_{n}^{+} \quad \text { if } \quad z_{n}^{+} \leq 5, \\
& v_{n}^{+}=5.0 \ln z_{n}^{+}-3.05 \quad \text { if } \quad 5<z_{n}^{+}<30, \\
& v_{n}^{+}=2.5 \ln z_{n}^{+}+5.5 \quad \text { if } \quad z_{n}^{+} \geq 30,
\end{aligned}
$$

where $v_{n}$ and $z_{n}$ represent the wall-normal velocity and coordinate, respectively. For the hydraulically rough bed, a modified log-law (cf. Ref. 19) is used according to

$$
v_{n}^{+}=2.5 \ln \frac{z_{n}}{k_{s}}+8.5
$$

where $k_{s}$ is the representative roughness height. The diameter of the sand particles is related to the roughness height $k_{s}$ as $k_{s} \approx 3 d_{90}$ (Ref. 20), in which $d_{90}$ is the particle diameter below which $90 \%$ of the particles (by weight) are smaller.

At the outflow boundary, a convective boundary condition is imposed. In order to specify appropriate inflow boundary conditions, a separate LES computation was performed of a straight open-channel flow over the topography that is present in the inflow region of the computational domain. In this area, the bed is nearly flat. For this separate 
TABLE I. Hydraulic parameters of the three simulation runs. $Q$ is the discharge, $V_{\text {av }}$ the bulk velocity, $H$ the mean water depth, $B$ the width of the channel, $k_{s}$ the roughness height of the bed, and Re the Reynolds number based on the bulk velocity and the mean water depth.

\begin{tabular}{lcccccc}
\hline \hline Run & $\begin{array}{c}Q \\
(1 / \mathrm{s})\end{array}$ & $\begin{array}{c}V_{\mathrm{av}} \\
(\mathrm{m} / \mathrm{s})\end{array}$ & $\begin{array}{c}H \\
(\mathrm{~m})\end{array}$ & $\begin{array}{c}B \\
(\mathrm{~m})\end{array}$ & $\begin{array}{c}k_{s} \\
(\mathrm{~m})\end{array}$ & $\operatorname{Re}(\cdots)$ \\
\hline $\mathrm{A}$ & 89 & 0.49 & 0.141 & 1.3 & 0.037 & 68000 \\
$\mathrm{~B}$ & 89 & 0.49 & 0.141 & 1.3 & $0.006-0.037$ & 68000 \\
$\mathrm{C}$ & 89 & 0.49 & 0.141 & 1.3 & 0.006 & 68000 \\
\hline \hline
\end{tabular}

LES computation, periodic boundary conditions were used in streamwise direction driving at the same Reynolds number as in the experiment.

The dynamic ripples moving along the bed are parametrized with an equivalent roughness height of $k_{s}=0.037 \mathrm{~m}$. This value consists of a contribution from the roughness of the sand particles themselves and a contribution from the unresolved bed forms and is equal to the $k_{s}$ value taken by Zeng et al. ${ }^{14}$ However, considering the speculative nature of this choice, the sensitivity of changes in this value is investigated by means of two additional simulations: one with $k_{s}=0.006 \mathrm{~m}$ and one with a $k_{s}$ value varying from $0.006 \mathrm{~m}$ at the most shallow point of the bed to $0.037 \mathrm{~m}$ at the deepest point of the bed.

Three simulations are performed: run A with $k_{s}=0.037 \mathrm{~m}$, run B with $k_{s}$ linearly varying from 0.006 to $0.037 \mathrm{~m}$, and run C with $k_{s}=0.006 \mathrm{~m}$. The hydraulic parameters of the three simulations are listed in Table I. All the three simulations are run on a grid containing $1248 \times 192$ $\times 72$ grid cells. Mesh independency issues for the used numerical code have already been discussed in Refs. 11 and 12 and in the Appendix. The 72 grid cells in the vertical direction are distributed over a total height equal to $0.4293 \mathrm{~m}$, yielding a regular vertical grid resolution of $6 \mathrm{~mm}$. Irrespective of the absence of measurement data on the ripple statistics, direct representation of the ripples would not be possible on this relatively coarse grid anyway. The three simulation runs with different wall-function characteristics will provide an indication of the sensitivity of the simulation outcome to the roughness height.

\section{Immersed boundaries}

Since a regular structured mesh is used, the way of treating the non-grid-conforming bed needs to be explained. For the representation of the bed topography, a modified version of the immersed boundary technique as proposed by Balaras $^{21}$ is adopted. In short, the applied method is as follows. The entire computational domain is subdivided in a fluid region (the area above the bed) and a solid region (the area containing the bed). Velocity points located in the fluid region which have at least one neighbor in the solid region are marked as boundary points. In direct forcing immersed boundary methods, the immersed boundary is imposed by manipulating the velocity according to some interpolation scheme, which is implicitly inherent to adding a body force to the equations of motion.

Usually, linear or quadratic interpolation schemes are

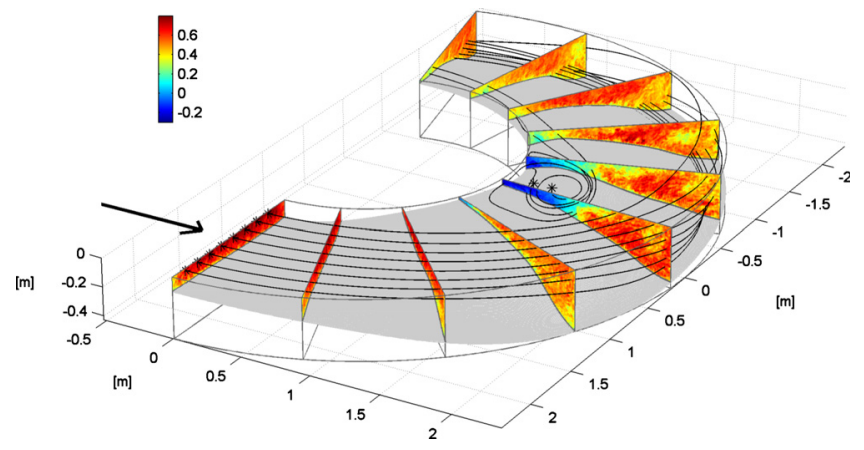

FIG. 3. (Color online) Instantaneous snapshot from run A of the turbulent streamwise velocities (in $\mathrm{m} / \mathrm{s}$ ) at some cross-sections in the curved part of the geometry and paths of particles that are released from locations signed with an asterisk. The arrow denotes the flow direction.

used at these boundary points. However, since in the present simulation of curved open-channel flow the bed is hydraulically rough, either linear or quadratic interpolation is physically improper. Namely, these kinds of interpolation are only valid if the boundary points are located in the viscous sublayer. In the present case, the distance between the most near-wall grid points and the bed itself is generally of the order of 0-120 wall units, thereby not sticking in any viscous sublayer. Therefore, Eq. (2) is used for this interpolation in order to mimic the proper near-bed physics in a very simple way. This modified version of the immersed boundary method of Balaras ${ }^{21}$ is explained more extensively in the Appendix.

\section{FLOW PHENOMENOLOGY}

\section{A. Primary flow, secondary flow, and recirculation}

In order to render the flow pattern along the bend, the instantaneous streamwise velocities for run A are shown in Fig. 3 at the cross-section at $0^{\circ}, 21^{\circ}, 43^{\circ}, 64^{\circ}, 86^{\circ}, 107^{\circ}$, $129^{\circ}, 150^{\circ}, 172^{\circ}$, and $193^{\circ}$ in the bend. Moreover, the particle paths of particles that are released at the locations denoted with an asterisk are shown in Fig. 3.

At the entry of the bend, the distribution of the streamwise velocities is skewed toward the inner bank due to the presence of the sudden favorable longitudinal pressure gradient as a consequence of the discontinuity in curvature, i.e., the change from straight flow to curved flow. In the upstream part of the bend, the flow decelerates as the flow becomes deeper (i.e., the cross-sectionally averaged bottom level is lower). Both the deepest point and the shallowest point in the flow are found around the $70^{\circ}$ cross-section, where the pool and the top of the pointbar are found.

Approximately halfway in the bend, around the $90^{\circ}$ cross-section, the main flow accelerates whereas return flow is found near the inner bank. This backflow is associated with a recirculation zone near the inner bank just downstream of the pointbar. This recirculation zone is clearly shown by the streamlines visualized by particle paths. Beyond about the $110^{\circ}$ cross-section, in the downstream part of the bend, the flow recovers from the strongly curvature in- 

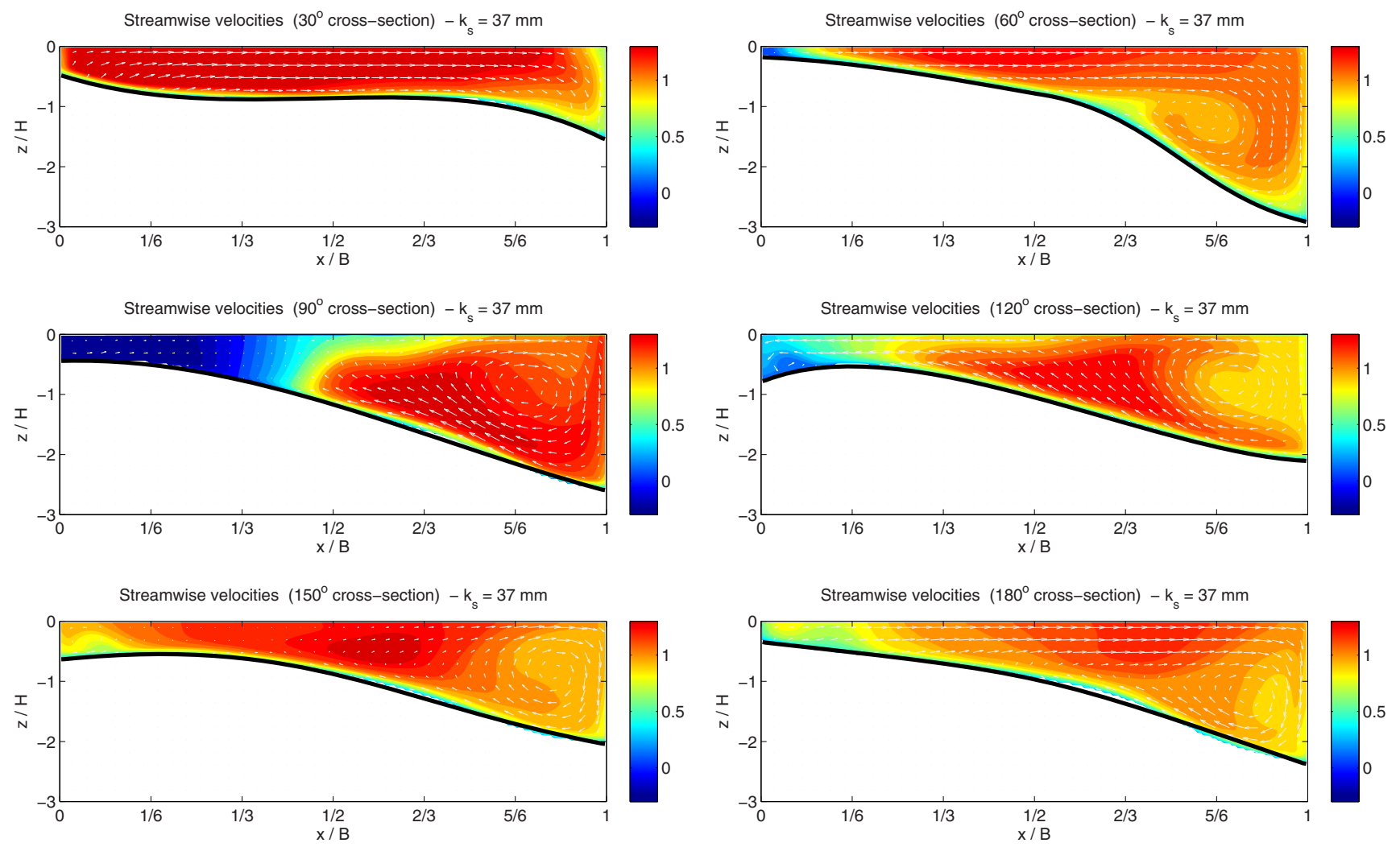

FIG. 4. (Color online) In lexicographic ordering: streamwise velocity patterns, from run A, at the cross-sections located at $30^{\circ}, 60^{\circ}, 90^{\circ}, 120^{\circ}, 150^{\circ}$, and $180^{\circ}$ in the bend. The values are made nondimensional with the bulk velocity $V_{\mathrm{av}}$. In the background of the pictures, the cross-sectional velocity vectors are shown at one out of five grid cells. From left to right denotes from inner bank to outer bank.

duced effects and the recirculation zone in the upstream part of the bend. This is also reflected by the bottom shape (also see Fig. 1), which is more gently arranged.

Although Fig. 3 shows the particle trajectories quite well, the subsequent analysis of the flow results needs a clear time-averaged picture of the flow behavior around the bend. Figure 4 provides such an image of the bend flow behavior by showing the pattern of the streamwise velocities at $30^{\circ}$, $60^{\circ}, 90^{\circ}, 120^{\circ}, 150^{\circ}$, and $180^{\circ}$ cross-section in the bend. Moreover, it shows the cross-sectional velocity vectors at each one out of five grid cells. The velocity patterns, shown for run A, show the strong spatial development of the flow in the upstream part of the bend $\left(30^{\circ}\right.$ and $60^{\circ}$ cross-sections), the recirculation zone at the inner bank $\left(90^{\circ}\right.$ cross-section), and the more mild flow evolution in the downstream part of the bend $\left(120^{\circ}, 150^{\circ}\right.$, and $180^{\circ}$ cross-sections).

Another view on the flow structure is provided by Fig. 5, where the vorticity and two-dimensional divergence are given for run A. The vorticity $\omega_{z}$, with the axis of rotation in vertical direction, and two-dimensional divergence $D_{z}$, in the horizontal plane, are defined as

$$
\omega_{z}=\frac{1}{r} \frac{\partial U}{\partial \theta}-\frac{1}{r} \frac{\partial V r}{\partial r} \quad \text { and } \quad D_{z}=\frac{1}{r} \frac{\partial U r}{\partial r}+\frac{1}{r} \frac{\partial V}{\partial \theta},
$$

respectively. The values of these quantities are made nondimensional by the mean water depth $H$ and the bulk velocity $V_{\mathrm{av}}$. Notice that the plots of the vorticity and the divergence also reflect the discontinuity in curvature, from straight to curved and from curved to straight, due to the additional cylindrical term. This is hence a physical phenomenon rather than a numerical artifact due to the coordinate change.

The vorticity at the surface, shown in the left panel of Fig. 5, clearly reveals the recirculation zone halfway in the bend near the convex inner bank of the flume. This region is denoted with the letter A. In this figure, the recirculation zone can be identified by a circular zone of counterclockwise rotating fluid with a diameter of about $0.5 \mathrm{~m}$.

At the interface between the main flow and the recirculation zone, a pronounced mixing layer is identified (region B). In Fig. 5, it is seen how the boundary layer, already visible in the straight inflow reach of the flume, separates from the inner bank at the highest point of the bottom, i.e., at the top of the pointbar. This is a rather logical location for the flow to separate because the bed topography itself gives rise to a change of sign in the longitudinal pressure gradient as the longitudinal gradient of the bed topography itself changes sign. After the boundary layer has detached from the inner bank, the resulting mixing layer develops spatially along a curved path due to the curvature of the main flow. In the picture of the vorticity, this region is indicated with a B; in the picture of the divergence this region develops along the locations assigned as $\mathrm{H}, \mathrm{I}$, and $\mathrm{J}$.

Another point of flow separation is found about $0.5 \mathrm{~m}$ downstream of the bend exit at point $\mathrm{C}$ in Fig. 5. At this point, a newly developed boundary layer detaches from the inner bank at the point where the water depth is, again, the smallest. The associated recirculation zone is, however, very weak. 

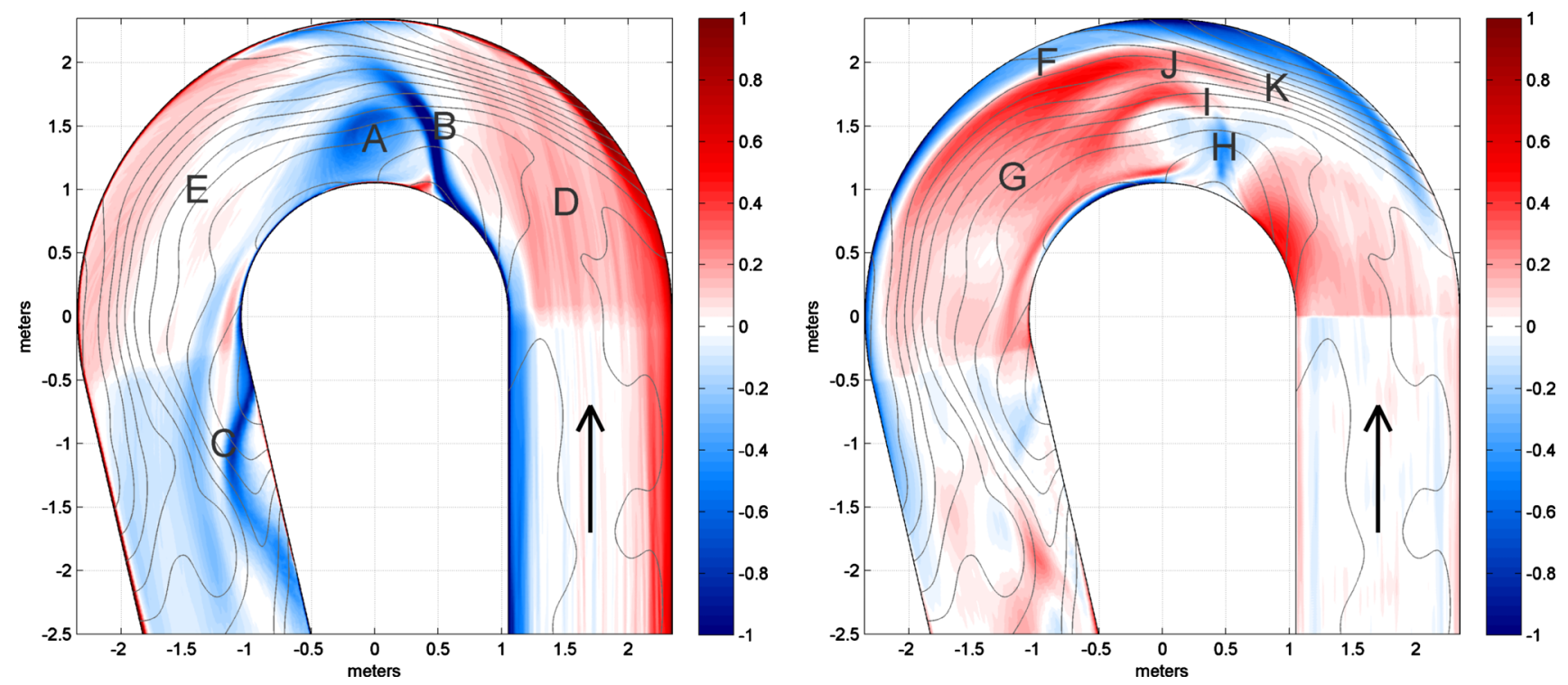

FIG. 5. (Color online) Vertical vorticity (left panel) and two-dimensional divergence in the horizontal plane (right panel) of the time-averaged velocity field from run A at the free surface. The values are made nondimensional using the mean water depth $H$ and the bulk velocity $V_{\text {av }}$. In the background of the figures, the topography of the bed is shown (cf. Fig. 1). The arrow denotes the flow direction.

The plot of the vorticity in the left panel of Fig. 5 also shows the strong spatial adaptation of the flow to the curvature of the flume in the upstream part of the bend (region D). In this area, the flow at the free surface rotates in clockwise direction. Around the $45^{\circ}$ cross-section the thickness of the boundary layer at the concave outer bank is very much confined due to the flow that impinges on the bank. At the downstream part of the bend, the boundary layer at the outer bank is very thin though, extending weakly but deeply into the core of the flow. In contrast with region $\mathrm{D}$, the downstream region $\mathrm{E}$ is an area of more quiescent flow: streamwise gradients of the bed topography as well as of the vorticity are small.

The right panel of Fig. 5 shows the two-dimensional divergence of the flow at the free surface and thereby the regions of upwelling $\left(D_{z}>0\right)$ and downwelling $\left(D_{z}<0\right)$ fluid motion. It is not surprising to see that near the outer bank the flow dives toward the bottom (region F). In the downstream region of the bend, the region of downwelling motion is rather narrow, whereas upwelling motion is observed over the larger part of the free surface (region G).

It is seen for the recirculation zone that both upwelling and downwelling occur in this area. This nicely reveals the strongly three-dimensional behavior of the flow, which is a net result of the superposition of secondary flow in the horizontal plane (i.e., recirculation) to the secondary flow in the cross-sectional plane (induced by the centripetal forces).

Along the curved mixing layer in the bend, downwelling motion is observed in its upstream part (location $\mathrm{H}$ ), whereas upwelling motion is found in its downstream part (locations I and J). Besides this, two regions of pronounced upwelling are identified. The first region starts at point $\mathrm{K}$ and extends toward the bend exit. The interface between this area of upwelling motion and the area of downwelling motion near the outer bank can be interpreted as a measure for the location of the center of the secondary flow cell in the cross-sectional plane.

The second region of pronounced upwelling starts at point I and stays aside the recirculation zone. In this region, the upwelling fluid motion has a strongly periodic nature. It was found from both a qualitative analysis (animations of the instantaneous velocity components) and a quantitative analysis (spectral analysis of the velocity fluctuations) (both not shown here) that the associated timescale of this oscillatory motion is about $1.5 \mathrm{~s}$. It was observed that about once per $1.5 \mathrm{~s}$, a coherent structure originates from the bottom and moves toward the free surface. We will see later on in this paper that this upwelling motion leaves a clear footprint in the pattern of the turbulent kinetic energy (tke).

\section{B. Comparison with experimental results}

For the $90^{\circ}$ cross-section, the three components of the velocity vector as these have resulted from the experiment and the large-eddy simulation (run A) are shown in Fig. 6. Notice that for the experimental result the quality of the data for $x / B<1 / 3$ is dubious since the water depth in this area is actually too shallow for the measurement device to be accurate.

In Fig. 6, it can be seen that at the $90^{\circ}$ cross-section the experimental results and numerical results are qualitatively very much alike. The streamwise velocities show a maximum of order $1.3 V_{\mathrm{av}}$ in the core region of the flow. The highest streamwise velocities in this cross-section are generally found close near the bottom. In the pool region of the cross-section, the agreement between experimental and numerical results is also qualitatively good.

However, a discrepancy is found for the vertical velocities near the outer bank. In this region, the downwelling 

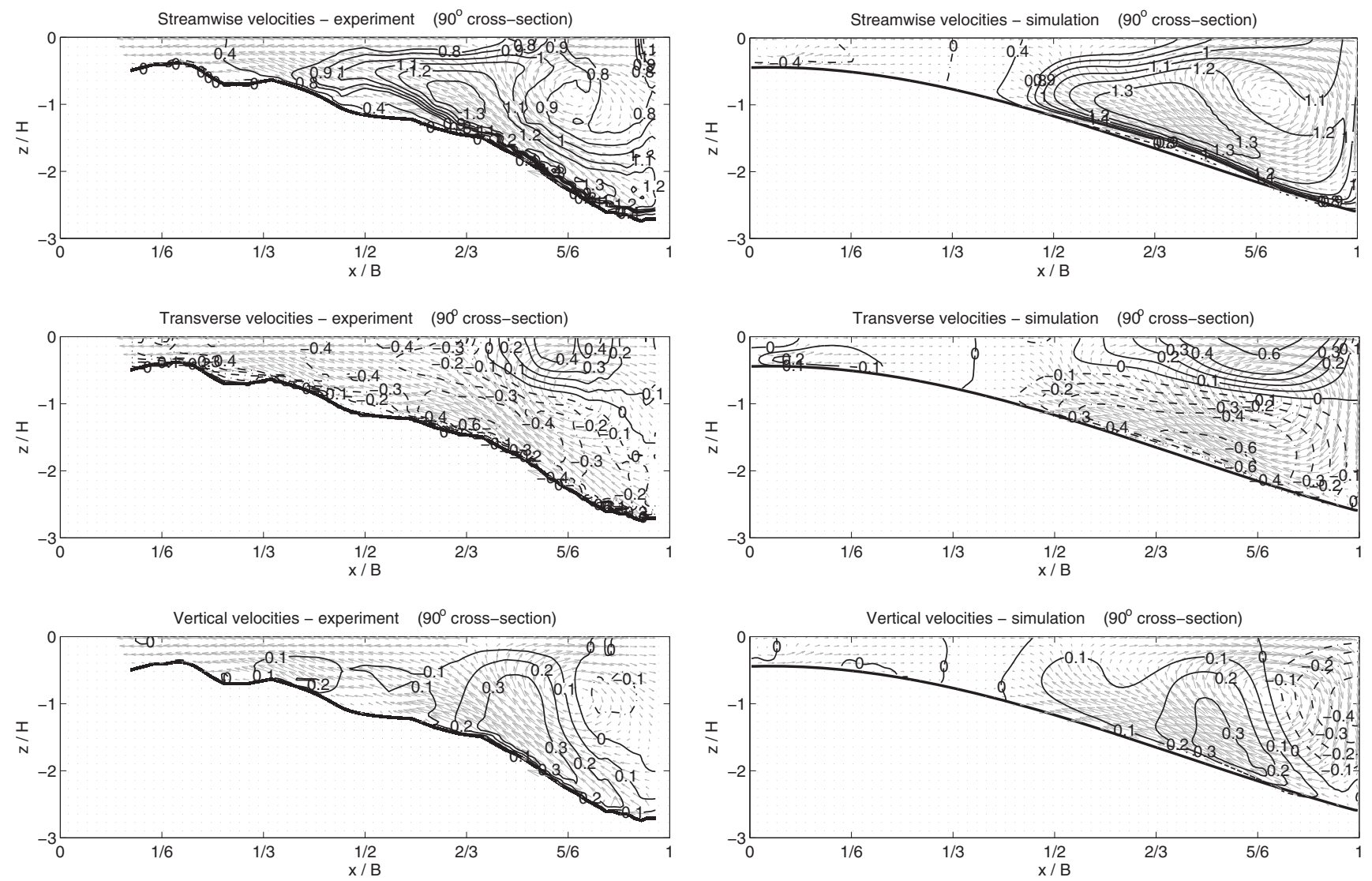

FIG. 6. Components of the velocity vector for the cross-section at $90^{\circ}$ in the bend. The values are made nondimensional with the bulk velocity $V_{\text {av }}$. From top to bottom: streamwise velocities, transverse velocities, and vertical velocities. Left panel: experimental results; right panel: numerical results. In the background of each picture, the vector plot of the cross-sectional flow pattern is shown. For the numerical results only one out of three vectors is shown in both directions. From left to right denotes from inner bank to outer bank.

motion is stronger in the LES results (about $0.4 V_{\text {av }}$ ) compared to the experimental results (about $0.1 V_{\mathrm{av}}$ ). This discrepancy is also reflected by the streamwise velocities. Near the outer bank, these are about $0.8 V_{\text {av }}$ in the experiment and are about $1.1 V_{\mathrm{av}}$ in the LES. Nevertheless, it seems that there is a kind of spatial lag between the LES results and the experimental results. Notwithstanding it shows an instantaneous snapshot, Fig. 3 already reveals that the pattern of the streamwise velocities at about the $84^{\circ}$ cross-section in the LES much resembles the pattern of the streamwise velocities as observed in the experiment at the $90^{\circ}$ cross-section (Fig. 6 , left upper plot). This could then suggest that the bed ripples, which are present in the experiment but absent in the LES computation, have a spatially delaying effect on the flow development along the bend. This is, however, quite speculative and difficult to verify because of the limited availability of the experimental data.

In the LES results, a clear footprint of the bed topography was found in the pattern of the transverse velocities. As already can be seen from Fig. 6 (right middle panel), the line of separation between outward directed and inward directed flow can be depicted as being in correspondence with the location where the pointbar ends and the pool begins. This correlation was found all over the upstream part of the bend, where the flow spatially develops. From this observation, it can be adduced that the flow just adapts to the prescribed topography by the redistribution of mass across the crosssection. This process has previously been specified in literature as topographic steering (cf. Refs. 13, 22, and 23).

In Fig. 7, the profiles of the velocities in streamwise and transverse direction are shown as these follow from the experiment and the LES computation. In addition, the results from the RANS computation are taken from the paper by Zeng et al. ${ }^{14}$ and shown in Fig. 7 as well. In this figure, it can be seen that the agreement between the experimental results and the LES results is variable. At some locations there is good agreement, at some locations not. Notwithstanding the moderate quantitative agreement, the qualitative agreement between the experiment and the LES computations is rather good.

Nonetheless, it can also be seen in Fig. 7 that the agreement between the LES computation and the RANS computation is very good. At some locations, the results from both simulation types perfectly coincide, whereas the experimental result deviates. The only profile at which the results from all the three approaches diverge is the profile of the transverse velocities at $x / B=5 / 6$ at the $60^{\circ}$ cross-section. At this cross-section, the transverse velocities from the LES and 

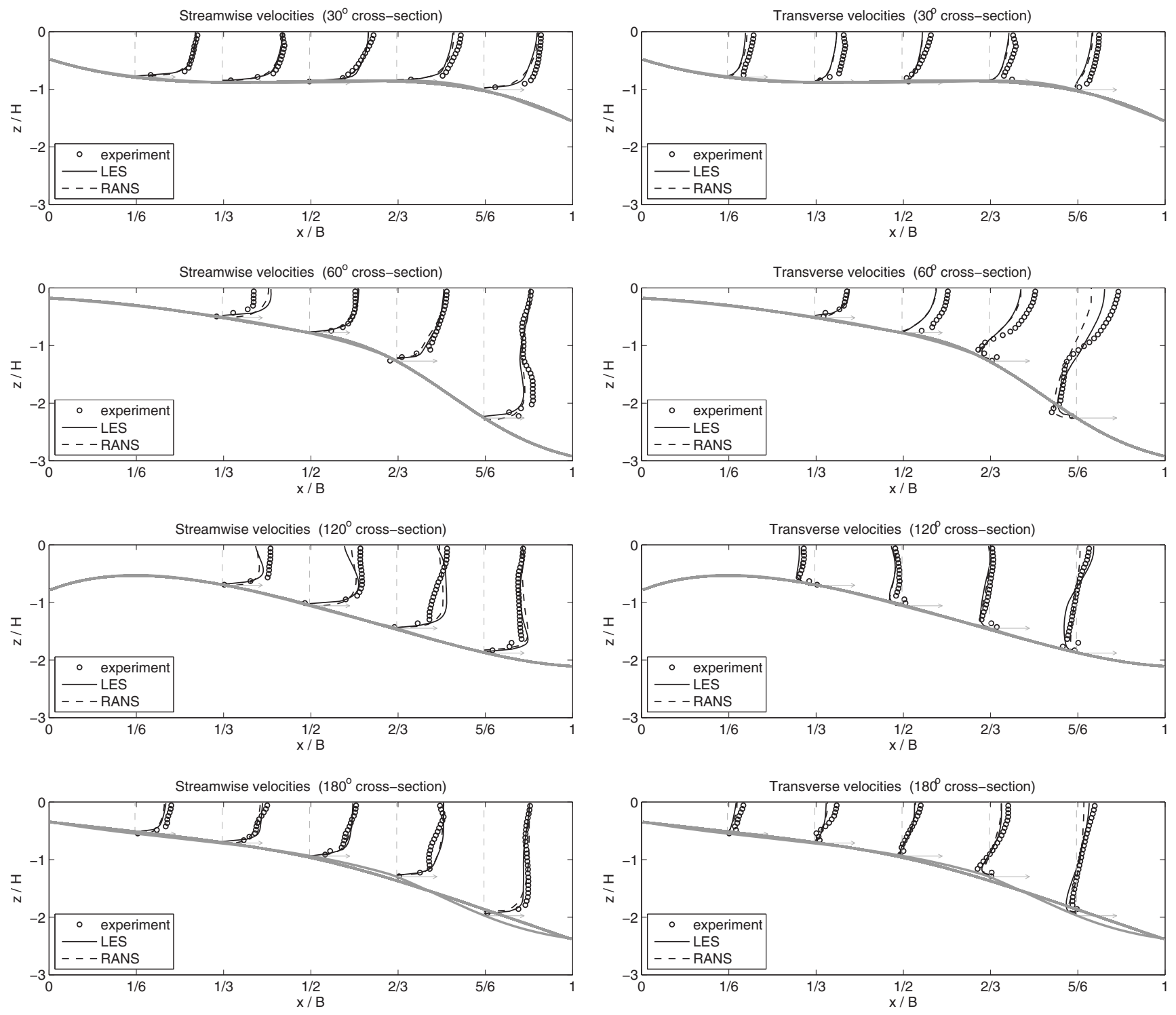

FIG. 7. Results from the experiment (circles), the LES computation for run A (solid lines), and the RANS computation (dashed lines) for the streamwise velocities (left panels) and the transverse velocities (right panels). From top to bottom: the $30^{\circ}$, the $60^{\circ}$, the $120^{\circ}$, and the $180^{\circ}$ cross-sections. The velocities are scaled with the bulk velocity $V_{\mathrm{av}}$. The arrow denotes a velocity equal to the bulk velocity. From left to right denotes from inner bank to outer bank.

RANS computations perfectly collapse at the locations $x / B=1 / 2$ and $x / B=2 / 3$, whereas the experiment indicates higher transverse velocities.

The differences between the experiment on the one hand and the LES and RANS computations on the other hand might be explained by the existence of the small-scale dunes of which the length scale of the height ranges from 1 to $10 \mathrm{~cm}$. In spite of the dense measuring grid, the spatial resolution was not sufficient to resolve the details of the dunes superposed on the macroscale topography. Thereby, the dune forms were not represented in the prescribed bed topography both in the LES and the RANS computation. As aforementioned, it can be conjectured that the existence of dunes has a spatially delaying effect on the flow development.

From the close agreement between the LES results and the RANS results it can be surmised that assumptions made within either the filtering (LES) or the statistical (RANS) framework are of marginal influence. Apparently, a simple modeling of the turbulence effects suffices. This issue will further be addressed in Sec. IV.

\section{Influence of roughness}

The results shown so far concern simulation run A. In run A, the small-scale dunes are parametrized by a roughness height $k_{s}=0.037 \mathrm{~m}$ (cf. Ref. 14). In order to investigate the sensitivity of the results to the choice of the roughness height $k_{s}$, results from run B (with $k_{s}$ varying from $0.006 \mathrm{~m}$ at the shallowest point to $0.037 \mathrm{~m}$ at the deepest point) and run $\mathrm{C}$ (with $k_{s}=0.006 \mathrm{~m}$ ) are shown.

Generally, it was found that the differences in the flow field between the different simulation runs were only marginal along the entire flume. In Fig. 8, the streamwise vorticity defined as 

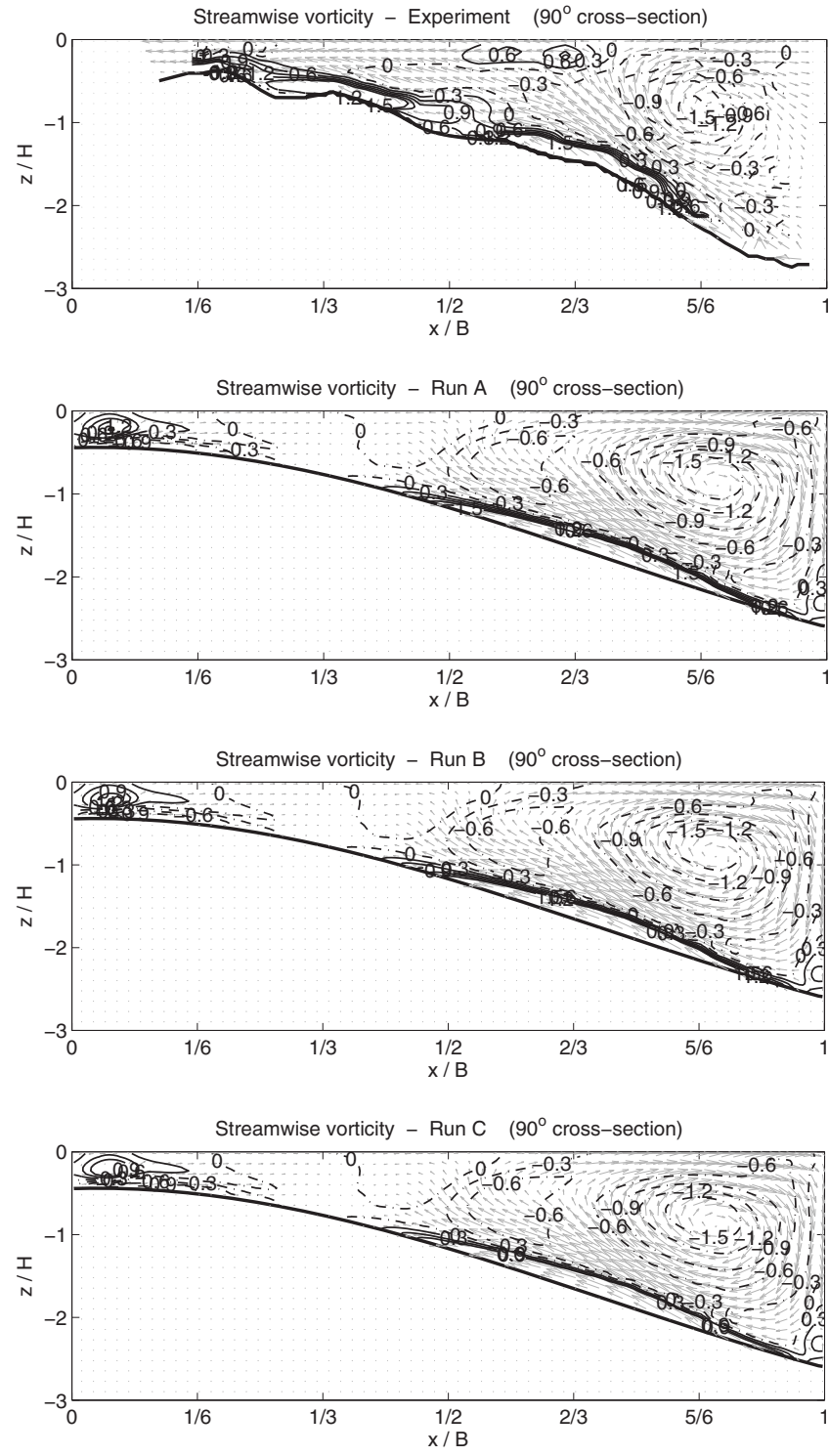

FIG. 8. Streamwise vorticity made nondimensional using the bulk velocity $V_{\text {av }}$ and the mean water depth $H$. From top to bottom: the experiment, run A, run $\mathrm{B}$, and run $\mathrm{C}$ for the $90^{\circ}$ cross-section. From left to right denotes from inner bank to outer bank.

$$
\omega_{\theta}=\frac{\partial W}{\partial r}-\frac{\partial U}{\partial z}
$$

at the $90^{\circ}$ cross-section is shown for the experiment as well as for all the three simulation runs. Figure 8 is shown to illustrate the small mutual deviations between the three simulation runs. It can be seen that the minimum value of -1.5 in the experiment is rather well captured by run A and run $\mathrm{B}$, but less well by run $\mathrm{C}$. The location of this local minimum slightly moves toward the inner bank if the bed roughness decreases.

Near the inner bank, there is a clear discrepancy observed in the rotational structure of the cross-sectional flow. In the experimental results, the flow at this location is pointed toward the inner bank, whereas in the numerical results the flow at this location is pointed toward the outer bank. As a result, the sign of streamwise vorticity is the opposite. This discrepancy can be understood from Fig. 5, where it can be seen that the $90^{\circ}$ cross-section is located more or less at the center of the recirculation zone (for run A). Therefore, small streamwise oriented changes in the location of the cross-section in the bend can easily result in a change in sign of the local secondary flow. The explanation for the oppositely directed flow in this area is thus explained by the fact that in the experiment the recirculation zone is located slightly more upstream compared to the simulations runs.

For the three simulation runs, it was found that the length of the recirculation zone slightly increases with decreasing roughness of the bed. The largest recirculation zone was found for run C: in this case its length is about $10 \%$ longer compared to run $\mathrm{A}$. This influence is also reflected in Fig. 8 in which it is shown that for run $\mathrm{C}$ the outward directed velocities are larger in the inner bank region compared to run $\mathrm{A}$.

The strength of the secondary flow along the bend can be investigated by means of the cross-sectional streamfunction. The secondary flow cell which can clearly be identified in the shown figures, e.g., Fig. 8, is generally considered as being the net result of centripetal forces, transversal pressure gradients, and the associated redistribution of momentum by advection. The pattern of this center region cell, also known as helical motion, can be rendered by means of the streamfunction $\psi$, which is defined as

$$
\psi=\frac{1}{2} \int_{r_{i}}^{r} W r \mathrm{~d} r+\frac{1}{2} \int_{z_{b}}^{z}-U r \mathrm{~d} z
$$

in which $U$ and $W$ represent the time-averaged transverse and vertical velocities, respectively. The variables $r_{i}$ and $z_{b}$ represent the curvature radius at the inner bank and the vertical coordinate of the bottom, respectively. The strength of the center region cell can simply be defined as max $\psi$. Considering the definition of $\psi$, scaling with the mean water depth $H$, the radius of curvature $R$ and the bulk velocity $V_{\mathrm{av}}$ is appropriate.

The development of $\max \psi$ along the bend is shown in Fig. 9. In this figure, it can be observed that the secondary flow strength increases with increasing roughness height. For each simulation run, the secondary flow strength increases monotonically from $35^{\circ}$ in the bend to a maximum value somewhere halfway in the bend. The location of this extremum moves downstream as the roughness of the bed decreases. This can be explained by the fact that the dimensions of the recirculation zone slightly increase with decreasing roughness height of the bed. However, the differences between the three simulation runs should not be overstated since the streamfunction $\psi$ is a rather sensitive quantity because of the enlarging effect on differences by the integration procedure. 


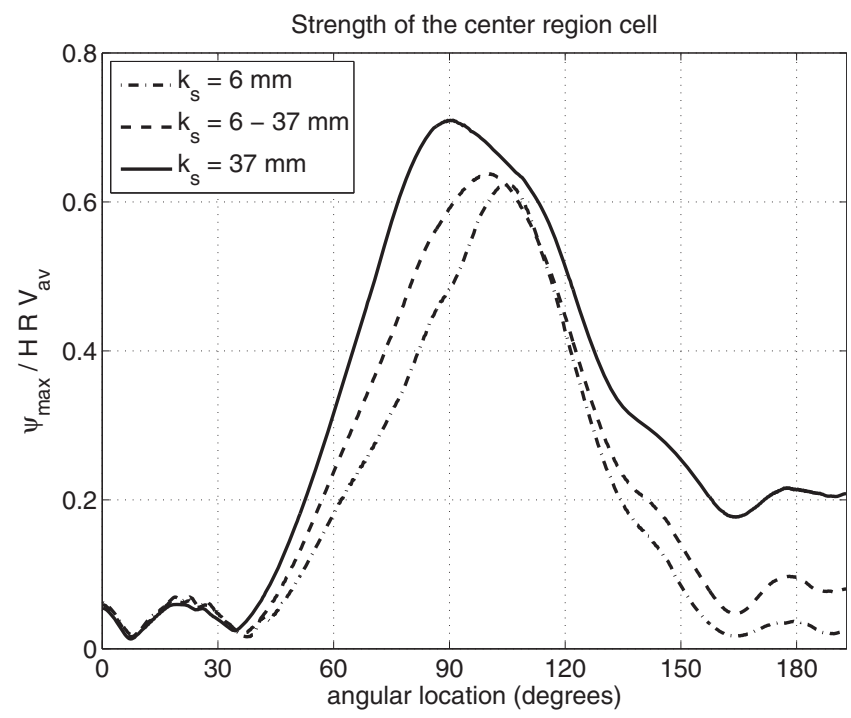

FIG. 9. Strength of the secondary flow for the three simulation cases with different bed roughness.

\section{TRANSPORT OF MOMENTUM}

\section{A. Momentum balance}

Recalling that $u$ and $v$ represent the transverse and streamwise velocities, respectively, Reynolds decomposition of the velocities is denoted as $u=U+u^{\prime}$ and $v=V+v^{\prime}$, in which the mean value is assigned with a capital and the fluctuating value is assigned with a prime. The momentum balance for the time-averaged transverse velocities $U$ and streamwise velocities $V$ are given by

$$
\begin{aligned}
& -\frac{\partial U}{\partial t}=U \frac{\partial U}{\partial r}+\frac{V}{r} \frac{\partial U}{\partial \theta}+W \frac{\partial U}{\partial z}-\frac{V^{2}}{r}, \\
& +\frac{\partial \overline{u^{\prime} u^{\prime}}}{\partial r}+\frac{1}{r} \frac{\partial \overline{u^{\prime} v^{\prime}}}{\partial \theta}+\frac{\partial \overline{u^{\prime} w^{\prime}}}{\partial z}+\frac{\overline{u^{\prime} u^{\prime}}-\overline{v^{\prime} v^{\prime}}}{r}, \\
& +\frac{\partial P}{\partial r}+\text { DIFFU }
\end{aligned}
$$

and

$$
\begin{aligned}
& -\frac{\partial V}{\partial t}=U \frac{\partial V}{\partial r}+\frac{V}{r} \frac{\partial V}{\partial \theta}+W \frac{\partial V}{\partial z}+\frac{U V}{r}, \\
& +\frac{\partial \overline{u^{\prime} v^{\prime}}}{\partial r}+\frac{1}{r} \frac{\partial \overline{v^{\prime} v^{\prime}}}{\partial \theta}+\frac{\partial \overline{v^{\prime} w^{\prime}}}{\partial z}+2 \frac{\overline{u^{\prime} v^{\prime}}}{r}, \\
& +\frac{1}{r} \frac{\partial P}{\partial \theta}+D I F F V,
\end{aligned}
$$

which obviously sum up to zero in case of statical steadiness. Notice that the density $\rho$ is incorporated in the pressure $p$ and that the terms $D I F F$-terms represent diffusion terms which are kept out of consideration.

Basically, four different types of budgets can be distinguished, namely, redistribution of momentum by advective processes, external forcing by centripetal effects, net trans- port of momentum by the Reynolds fluxes, the nonlinear adaptation to the velocity field by the pressure, and diffusion of momentum by viscous effects.

\section{B. Transport budgets}

The mechanisms of the transport of momentum can be deciphered by a term-by-term analysis of the momentum equations. For this purpose, the focus is restricted to streamwise momentum and transverse momentum for the simulation run A. Generally, it was found that transport by turbulence stresses is negligible as well as diffusion of momentum. What remains is a balance of the advective forces, centripetal forces, and pressure gradient forces.

As aforementioned, the flow can be divided in a strongly spatially developing upstream region (between $\sim 0^{\circ}$ and $80^{\circ}$ ) and a more quiescent flowing downstream region (between $\sim 120^{\circ}$ and $180^{\circ}$ ) where streamwise gradients in the bed topography are rather small. The latter region in general and the $135^{\circ}$ cross-section in particular is chosen for the termby-term analysis of the momentum transport equations. The five dominant terms of the momentum balance, both in streamwise and in transverse directions, are shown in Fig. 10. In the left panels of the figure the budgets of the transverse momentum balance are shown, whereas in the right panels of the figure the budgets of the streamwise momentum equations are shown.

By just summing up the five budgets, it can be checked that indeed the Reynolds contributions and the diffusion contributions are not needed to close the balance. The relative unimportance of the turbulence fluxes as regards the transport of momentum explains why the results from the LES and the RANS computations hardly differ (see Fig. 7). This implies that the choice for the closure of the Reynolds stress tensor (in case of a RANS computation) or the subgrid stress tensor (in case of a LES computation) is not important. Apparently, the flow is fully dominated by centripetal effects and topographic steering.

Considering the advective transport of transverse momentum (upper three left panels in Fig. 10), it can be seen that the cross-sectional contributions (i.e., in $r$ and $z$ directions) are the highest in the pool near the center of the secondary flow cell in the outer bank region, whereas the streamwise contribution (i.e., in $\theta$ direction) is the highest in the pointbar region of the cross-section. It is not surprising that the centripetal contribution $-V^{2} / r$ and the pressure gradient contribution are rather pronounced, since the curvature of the flume is quite strong.

The pattern of the advective transport of streamwise momentum (upper three right panels in Fig. 10) shows similar patterns, i.e., relatively large values of the transverse and vertical components in the pool (be it smaller compared to the transverse momentum transport) and large values of the streamwise component in the pointbar region. However, large contributions are found near the bed since the gradients of the streamwise velocities are quite large there. The interaction between the transverse velocities and streamwise velocities, represented by the $U V / r$-term, is rather weak as well as the contribution of the streamwise pressure gradient. 

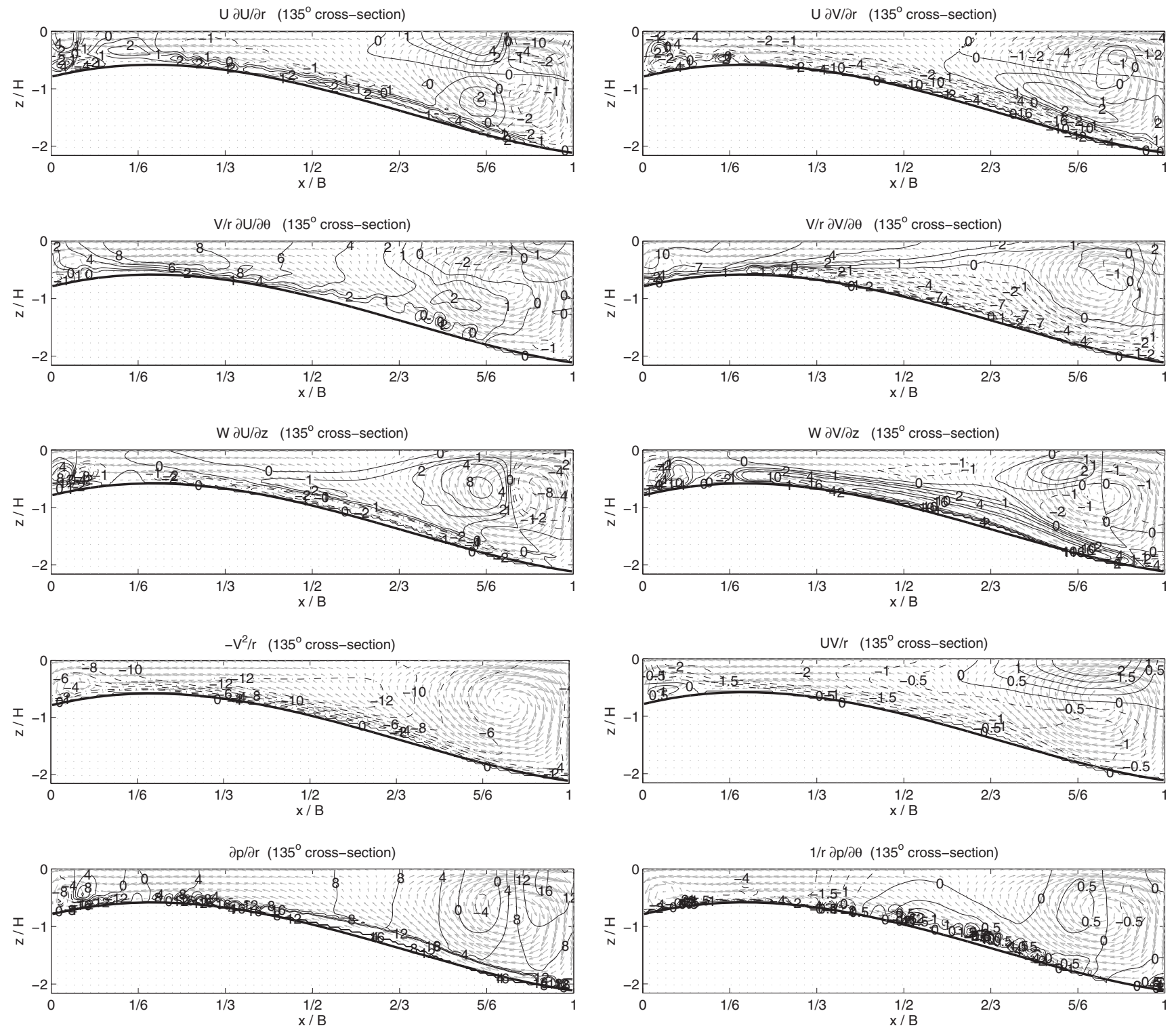

FIG. 10. Dominant budgets of the balance equations for transverse momentum (left panels) and streamwise momentum (right panels). The values are made nondimensional using the mean water depth $H$ and the bulk velocity squared $V_{\text {av }}$.

Generally, it was found that the pattern of the momentum transport as observed at the $135^{\circ}$ cross-section is representative of the general pattern in the downstream area of the flow, both in magnitude and in distribution. The distribution of the momentum transport contribution is also very similar in the upstream region, but in this region the magnitude of the contribution is about 50\% higher due to the strongly spatially developing nature over the flow in this region.

\section{TURBULENCE STRUCTURE}

\section{A. Turbulent kinetic energy}

Although gradients of the Reynolds stresses are apparently not significant within the context of transport of momentum, the Reynolds stresses themselves are relevant for the proper understanding of the flow characteristics. Moreover, turbulence shear stresses are known to be closely re- lated to changes in the bed forms. Therefore, an analysis of the turbulent kinetic energy and the turbulence structure is worthwhile.

The tke, defined as

$$
k=\frac{1}{2} \overline{u^{\prime 2}}+\frac{1}{2} \overline{v^{\prime 2}}+\frac{1}{2} \overline{w^{\prime 2}},
$$

is shown for run A in Fig. 11. In this figure, the tke is shown at the same locations as the instantaneous velocity profiles were shown in Fig. 3. The values are made nondimensional using the bulk velocity squared $V_{\mathrm{av}}^{2}$.

In Fig. 11, it can be seen that in the upstream part of the bend, an eruption of tke occurs. The pronounced local increase of tke gains strength in the area halfway in the bend and weakens in the downstream part of the bend. In this part of the bend, a local extremum of tke is found near the outer 


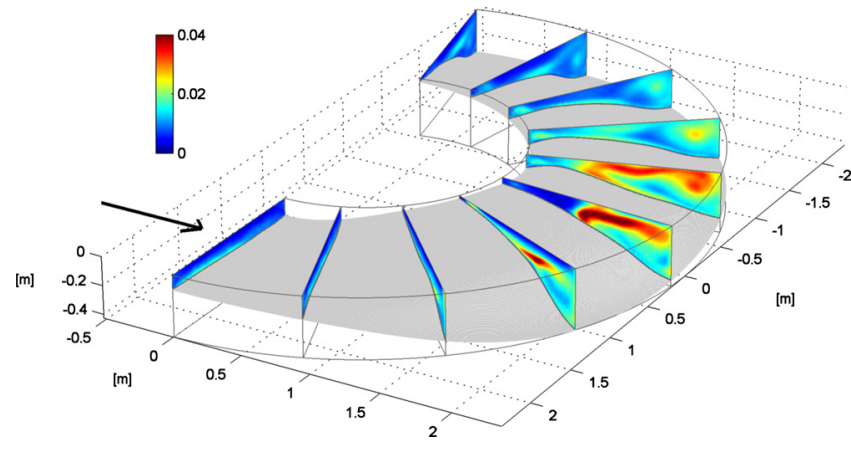

FIG. 11. (Color online) Turbulent kinetic energy for run A scaled with the bulk velocity squared $V_{\mathrm{av}}^{2}$.

bank in the core of the secondary flow cell. At the inlet of the bend, the highest tke values are obviously found near the bed.

In order to better render the mechanisms behind the pattern of the tke, its production is considered, denoted by $P$, and given by

$$
P=-\frac{1}{2}\left(\overline{u_{i}^{\prime} u_{j}^{\prime}}-\frac{2}{3} k \delta_{i j}\right) \overline{S_{i j}},
$$

with $i, j=r, \theta, z, \delta_{i j}$ the Kronecker delta and $\overline{S_{i j}}$ the rate-ofstrain tensor, based on the mean velocities, containing the components

$$
\begin{aligned}
& \overline{S_{r r}}=2 \frac{\partial U}{\partial r}, \\
& \overline{S_{\theta \theta}}=2\left(\frac{1}{r} \frac{\partial V}{\partial \theta}+\frac{U}{r}\right), \\
& \overline{S_{z z}}=2 \frac{\partial W}{\partial z}, \\
& \overline{S_{\theta r}}=\overline{S_{r \theta}}=\frac{\partial V}{\partial r}+\frac{1}{r} \frac{\partial U}{\partial \theta}-\frac{V}{r}, \\
& \overline{S_{\theta z}}=\overline{S_{z \theta}}=\frac{1}{r} \frac{\partial W}{\partial \theta}+\frac{\partial V}{\partial z}, \\
& \overline{S_{z r}}=\overline{S_{r z}}=\frac{\partial U}{\partial z}+\frac{\partial W}{\partial r} .
\end{aligned}
$$

Patterns of the production of tke are given in Fig. 12. The values in this figure are scaled by means of the mean water depth $H$ and the bulk velocity cubed $V_{\mathrm{av}}^{3}$. In the background of the plots, the velocity vectors of the cross-sectional flow field are shown.

A more detailed analysis of the data resulted in the insight that the location of the tke eruption can first be found around $45^{\circ}$ in the bend. The peculiarity of this cross-section is that this is the first cross-section where significantly upwardly oriented vertical velocities occur. As aforementioned, the flow is mainly driven by topographic steering, which basically means that the bottom topography acts like a mold to the flow. As a consequence, the sign of the cross-sectional velocities is dictated by the bed topography: transverse ve-
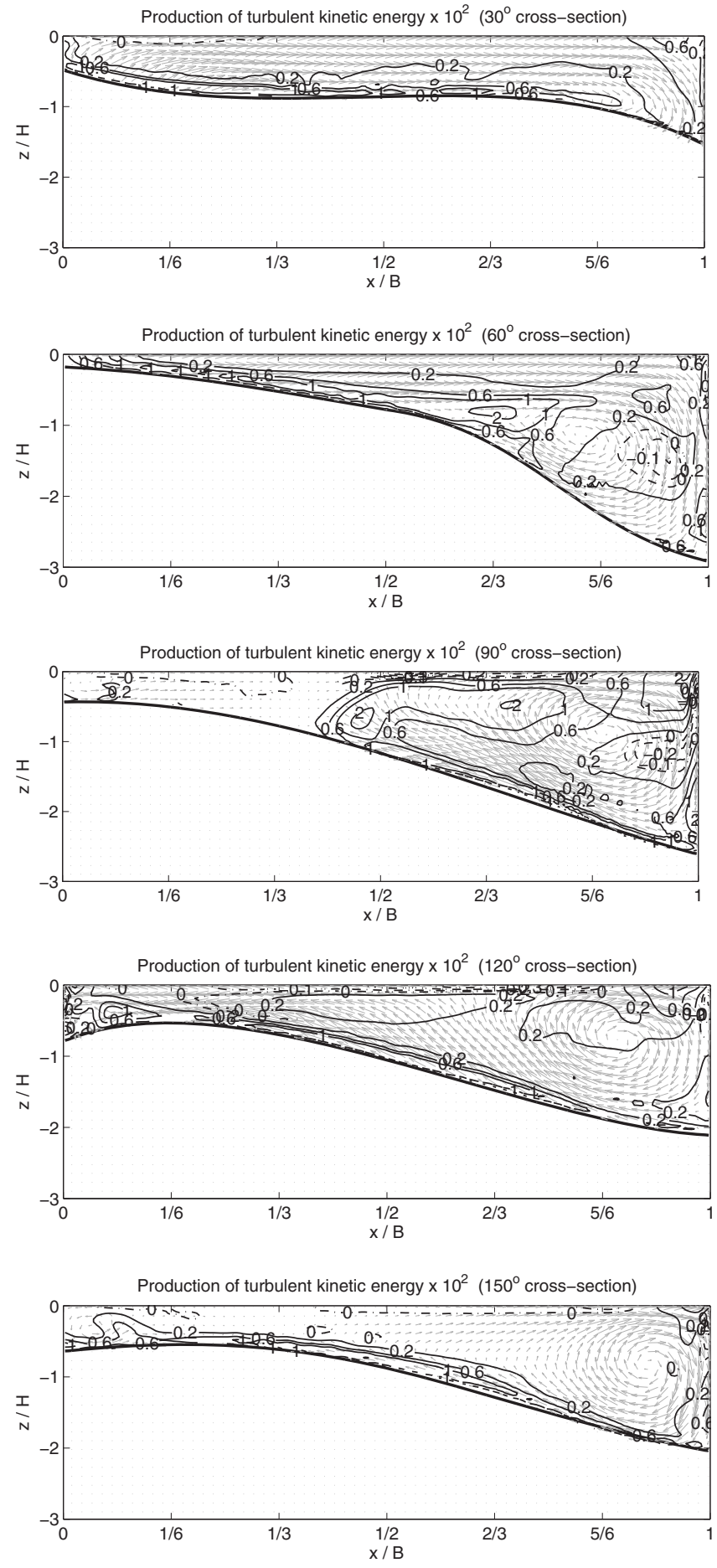

FIG. 12. Production of turbulent kinetic energy made nondimensional by means of the mean water depth $H$ and the bulk velocity cubed $V_{\mathrm{av}}^{3}$. From top to bottom: the $30^{\circ}, 60^{\circ}, 90^{\circ}, 120^{\circ}$, and $150^{\circ}$ cross-sections.

locities directed toward the inner bank are found at $20^{\circ}$ for the first time, whereas vertical velocities directed toward the free surface are found at $45^{\circ}$ for the first time. This flow behavior can also be recognized in the velocity vector plots shown in the background of Fig. 12.

In the figures of the tke production at $60^{\circ}$ and $90^{\circ}$, shown in Fig. 12, it can be seen that the production of tke has a pronounced peak near the bottom, in the center region of the cross-section. The velocity vectors in the background 
show that indeed this eruption of tke is found at the location where upwelling fluid motion collides with the outward directed secondary flow stream at the $60^{\circ}$ cross-section and at the location where the upwelling fluid motion collides with the quiescent recirculation flow region at the $90^{\circ}$ crosssection. In the other panels shown in Fig. 12, such a collision is absent and the production of tke has its maximum near the bottom where it is usually found. This upwelling motion was already identified in Fig. 5 where a belt of positive $D_{z}$ is seen starting from point I.

Roughly, it is found that the spatial distribution of the tke production is the same as of the tke itself. Nonetheless, areas of weak backscatter of tke from the turbulent motion to the mean motion (within the resolved part of the energy density spectrum) are found near the outer bank at the $60^{\circ}$ and $90^{\circ}$ cross-sections.

\section{B. Bed shear stresses}

The bed shear stresses are a measure for the forces that are exerted on the bed and are relevant within the context of erosion and deposition of sand particles at the bed. The bed shear stresses are defined as the friction velocity squared, denoted as $v_{*}^{2}$. In order to nondimensionalize the bed shear stresses, $v_{*}^{2}$ is scaled with the bulk velocity squared $V_{\mathrm{av}}^{2}$, thus obtaining the friction coefficient $c_{f}$. The wall function is applied on the total velocity vector based on the wall-parallel velocity components.

The distribution of the friction coefficient $c_{f}$ is, being multiplied by $10^{3}$, shown in Fig. 13 for run A (with $k_{s}=0.037 \mathrm{~m}$ ) in the upper panel, run B (with $k_{s}$ ranging from 0.006 to $0.037 \mathrm{~m}$ ) in the middle panel, and run $\mathrm{C}$ (with $\left.k_{s}=0.006 \mathrm{~m}\right)$ in the lower panel. Notice that the colorbar used in each subplot is different for each simulation run. The largest friction coefficients are obviously found for run A, since for this case the roughness height is the largest.

Nonetheless, the distribution of the friction coefficient is more or less the same for each case. At the entry of the bend, the largest friction is experienced near the inner bank, since in this region the velocities are the highest as a result of the strongly favoring pressure gradient due to the curvature discontinuity. Small friction coefficients are found on the pointbar, and in the recirculation zone just behind the pointbar as in this area, the fluid moves slowly.

Large friction is also experienced in the pool region of the bend, about halfway in the bend. It is remarkable that for run $\mathrm{C}$ (with $k_{s}=0.006 \mathrm{~m}$ ) the friction coefficient is relatively small compared to the values at the entry of the bend in comparison to run A and run B. In the downstream part of the bend, the bed friction is moderate and relatively equally distributed. In the result for run A, however, a spot of relatively large bed friction is found in the area around the $150^{\circ}$ cross-section.

Another remarkable thing is that run $\mathrm{C}$ (lower panel in Fig. 13) shows a clear footprint of the upwelling belt of flow as it is also recognized for run A (right panel of Fig. 5) and in the analysis of the structure of the turbulence kinetic energy (Fig. 11). The lower panel of Fig. 13 clearly shows how halfway in the bend an elongated patch of high values of $c_{f}$ is
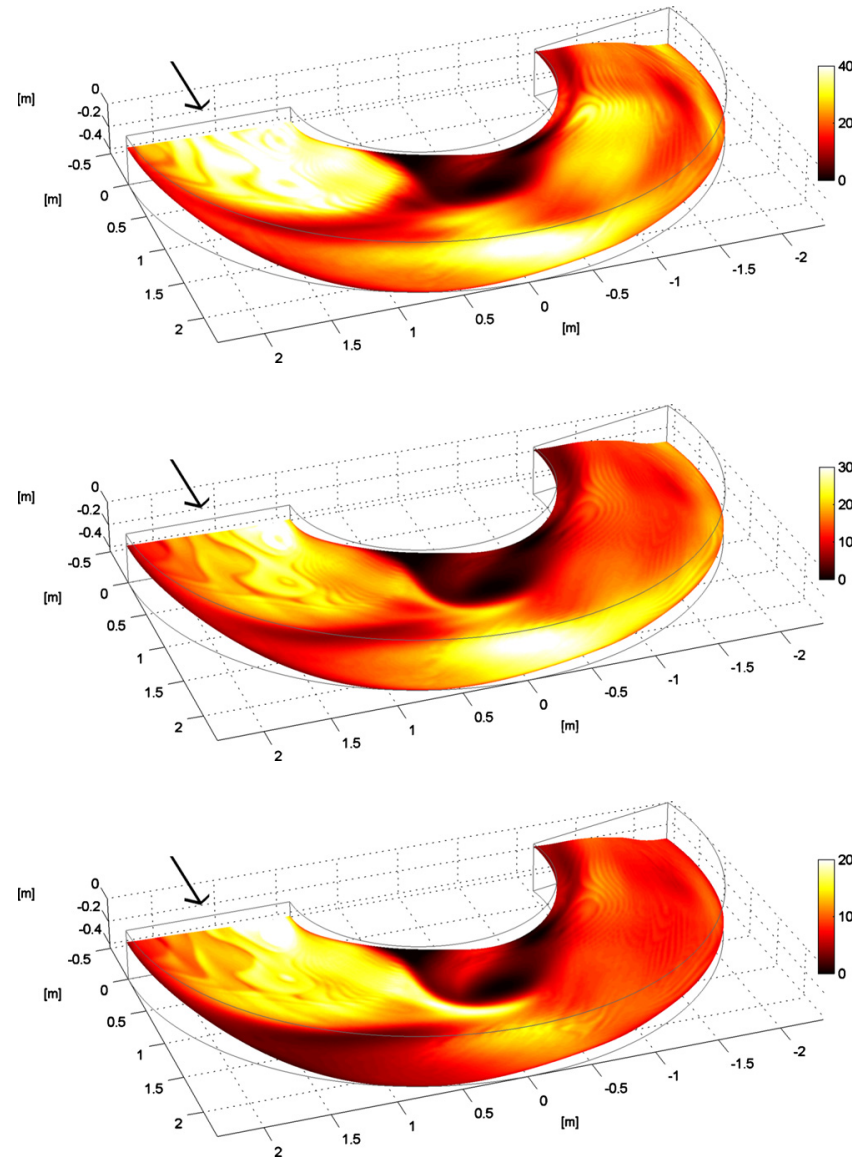

FIG. 13. (Color online) Bed shear stresses, given as $c_{f} \times 10^{3}$, for run $\mathrm{A}$ (upper panel), run B (middle panel), and run C (lower panel). The arrow denotes the flow direction.

found exactly between the pointbar region (with small $c_{f}$ values) and the pool region (with high $c_{f}$ values).

\section{Turbulence anisotropy}

The structure of the turbulence can further be studied by means of the normalized anisotropy tensor $b_{i j}$ which is defined as

$$
b_{i j}=\frac{\overline{u_{i}^{\prime} u_{j}^{\prime}}}{\overline{u_{k}^{\prime} u_{k}^{\prime}}}-\frac{1}{3} \delta_{i j} .
$$

Because the trace of this tensor $b_{i j}$ is zero, it has only two independent invariants, denoted as $\xi$ and $\eta$. These two invariants are related to two eigenvalues, $\lambda_{1}$ and $\lambda_{2}$, by (see Ref. 24)

$$
\begin{aligned}
& \eta^{2}=\frac{1}{3}\left(\lambda_{1}^{2}+\lambda_{1} \lambda_{2}+\lambda_{2}^{2}\right), \\
& \xi^{3}=-\frac{1}{2} \lambda_{1} \lambda_{2}\left(\lambda_{1}+\lambda_{2}\right) .
\end{aligned}
$$

These invariants $\xi$ and $\eta$ can graphically be shown in an anisotropy invariant map (see Ref. 25), often referred to as the Lumley triangle. A detailed interpretation of the Lumley triangle is given by Ref. 26 .

The values of $\eta$ and $\xi$ are evaluated at four different cross-sections: a cross-section at $2.2 \mathrm{~m}$ upstream of the bend (in fact, just straight channel flow), a cross-section at $60^{\circ}$ in 

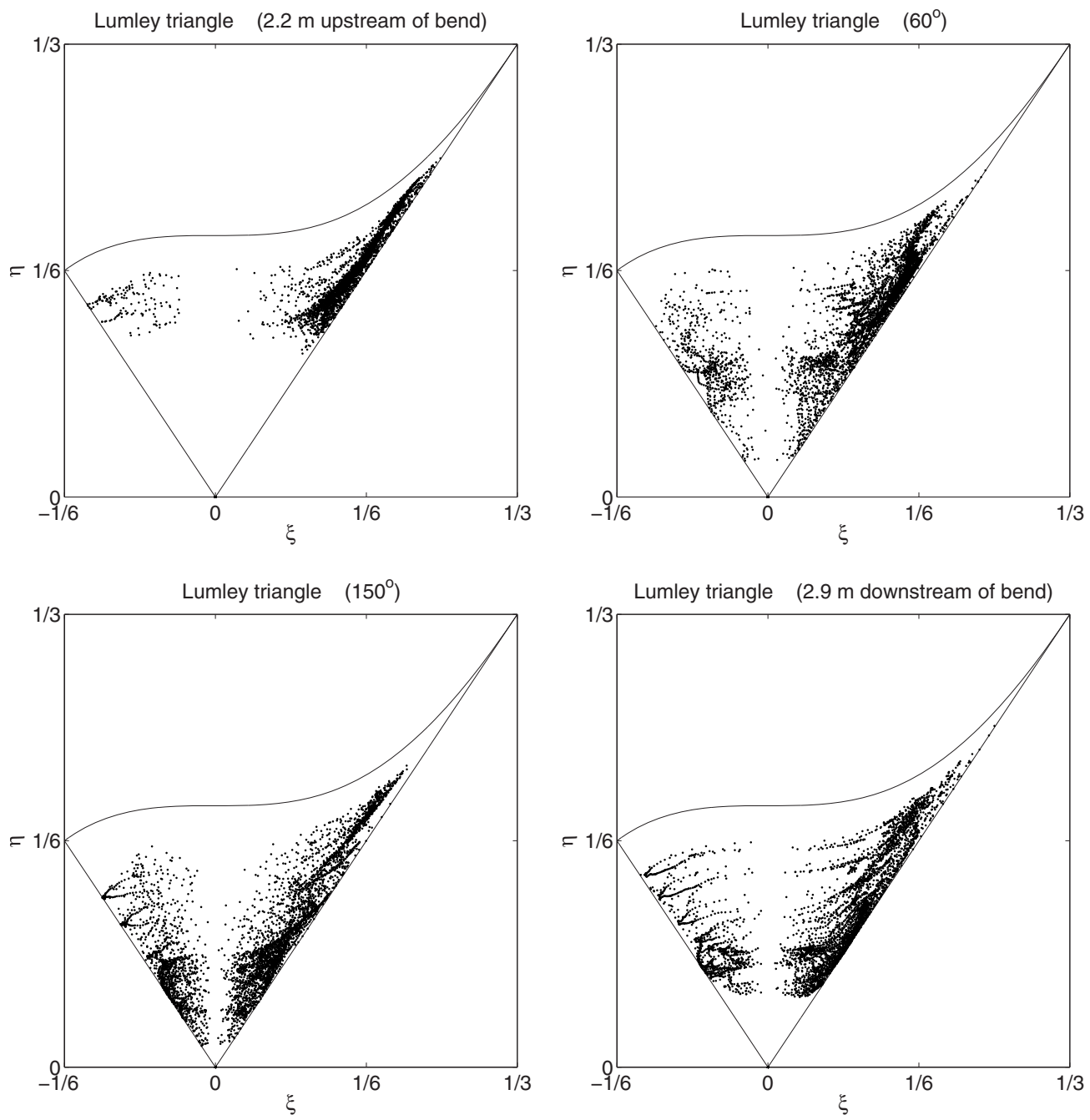

FIG. 14. Lumley triangles at four different cross-sections along the flume. In lexicographic ordering: the cross-section at $2.2 \mathrm{~m}$ upstream of the bend, the cross-section at $60^{\circ}$, the cross-section at $150^{\circ}$, and the cross-section $2.9 \mathrm{~m}$ beyond the bend exit.

the bend (flow is strongly spatially developing), a crosssection at $150^{\circ}$ (flow is spatially rather well developed), and a cross-section at 2.9 beyond the bend exit (flow is recovering from centripetal effects and returns to straight flow again). The Lumley triangles for these four cross-sections are shown in Fig. 14.

It is known for straight boundary layer flow that the $\eta, \xi$ values are found near the right straight side of the triangle, which represents axisymmetric turbulence with one large eigenvalue (cf. Ref. 27). This type of behavior is recognized in the picture of the cross-section $2.2 \mathrm{~m}$ upstream of the bend. The tendency of the $\eta, \xi$ values toward the curved upper side of the triangle indicates a tendency toward two-dimensional turbulence. The presence of this tendency is explained by the high aspect ratio of the cross-section, $B / H \approx 9$.

In the bend, there is a strong tendency visible toward the origin of the triangle, which is associated with isotropic turbulence. Particularly at the $150^{\circ}$ cross-section, the Reynolds stress tensor appears to be rather isotropic. Beyond the bend, in the straight outflow reach, the turbulence structure retreats from its rather isotropic state, be it rather slowly: still at the cross-section at $2.9 \mathrm{~m}$ downstream of the bend exit, the bend influences are quite strong.
The strong tendency toward isotropic turbulence is interesting from a turbulence modeling point of view. In Sec. III, it was visualized that the model results from both the LES computation and the RANS computation hardly differ from each other. In Sec. IV, it was shown that this similar behavior could be explained by the fact that the contributions of the Reynolds stress gradients to the transport of momentum are minimal. Figure 14 now shows in addition that the turbulence structure tends to be more or less isotropic, which is the basic assumption of many RANS models in general and the model of Zeng et al., ${ }^{14}$ who have performed the RANS computation, in particular.

\section{SUMMARY}

Large-eddy simulation was used to investigate the curved open-channel flow over complex bed topography based on the physical laboratory experiment by Blanckaert. ${ }^{13}$ The bed topography, as it has developed in the experiment, contains a pointbar in the inner bank region of the bend, a pool in the outer bank region of the bend, and small-scale turbulence induced dunes. In spite of the dense measuring grid, the spatial resolution was, however, not sufficient to 
resolve the details of the dunes superposed on the macroscale topography. Therefore, the focus behind the present LES computations was on the influence of the large-scale topography of the bed while parametrizing the small-scale dunes in terms of bed roughness.

Three separate LES computations were run with a different roughness height of the bottom. It was found that the differences between these three simulations are rather small as regards the primary flow structure. Moreover, it was found for all the three cases that the flow structure is fully determined by the large-scale bed topography, a process that has previously been depicted in literature as topographic steering (cf. Refs. 13, 22, and 23). The results from the LES show qualitative good agreement with the experimental findings. Quantitative differences are explained by the existence of the small-scale dune forms which are not directly accounted for in the LES due to the coarse measurements of the bed topography. It is conjectured that the existence of dunes on the bed effectuates a spatial lag in the streamwise development of the flow.

The flow pattern in general was found to be very complex due to the combined influence of a complex bed topography and strong curvature of the flume. Just downstream of the shallow pointbar area, a pronounced recirculation zone was found that spans about half of the width of the flume. At the interface of the recirculation zone and the main flow, a distinct mixing layer was observed, which is the result of separation of the upstream boundary layer from the convex inner bank on top of the pointbar. On this interface, strong upwelling flow motion was observed, which is accompanied by large production of turbulent kinetic energy. Because of the confinement of the cross-section due to the presence of the recirculation zone, the secondary flow strength was found to be the strongest halfway in the bend.

As regards the bed shear stresses, it was found that the bed suffers from the strongest flow attack at the entry of the bend where the flow is relatively shallow and in the pool region of the bend where the flow is relatively deep. By changing the roughness height of the bed, mainly the magnitude of the bed shear stresses is influenced rather than its distribution.

Differences between the present LES computation and the RANS computation carried out by Zeng et al. ${ }^{14}$ were found to be marginal for the larger part of the flow. The good mutual agreement is explained by the observation that turbulence related momentum transport does not play a role in the complete pattern of the momentum transport, which is due to the combination of strong curvature and the presence of a complex topography. Moreover, it was found that in the bend, there is a strong tendency toward isotropy of the turbulence stresses.

With these conclusions, the present paper has presented new insights into the physics of curved open-channel flows over topography. First, it is remarkable that the turbulence structure of the flow tends toward isotropy, whereas anisotropy might well be expected given the highly complex nature of the geometry. From a modeler's point of view, this tendency is beneficial as regards the reduction of the computational requirements, since an isotropic turbulence model could be chosen within the RANS context for the kind of flows considered in this paper. Second, irrespective of the isotropic nature of the turbulence, it has appeared that transport of momentum by the Reynolds stresses is negligible. This conclusion is in line with the idea of topographic steering as addressed by Refs. 13, 22, and 23. However, this issue is rarely addressed in literature, and we are the first ones who prove that this is the case for the current experiment. Third, we also elucidate the emergence of coherent structures appearing on the complex three-dimensional mixing layer at the interface of the recirculation zone near the inner bend and the main flow in the outer bend. This is not possible using a RANS computation and illustrates the added value of LES. It appears that much turbulent kinetic energy is produced in this area and that the coherent structures have a dominant timescale of about $1.5 \mathrm{~s}$. Besides these three physical aspects, the paper moreover illustrates the ease and success of the combination of a very easy to implement immersed boundary method with a simple wall model that has been incorporated in the code, a combination that is used very rarely.

\section{ACKNOWLEDGMENTS}

This work is supported by the Dutch Technology Foundation (STW), applied science division of NWO, and the Technology Programme of the Ministry of Economic Affairs.

\section{APPENDIX: COMPLEX GEOMETRIES IN STRUCTURED REGULAR GRIDS}

\section{The immersed boundary method (IBM)}

The IBM that is implemented in our Navier-Stokes solver is based on the method that is proposed by Balaras. ${ }^{21}$ Immersed boundary methods, in general, are based on the concept that the embedded solid boundary can be represented by a body force following

$$
\frac{u_{i}^{n+1}-u_{i}^{n}}{\Delta t}=R H S_{i}+f_{i},
$$

with $R H S_{i}$ incorporating the pressure term, the advective terms, and the diffusive terms. If on a certain timestep $n+1$ at a location $i$, the desired velocity equals $V_{i}^{n+1}$, then, obviously, the body force can be computed following

$$
f_{i}=-R H S_{i}+\frac{V_{i}^{n+1}-u_{i}^{n}}{\Delta t}
$$

Suppose that a certain solid boundary $\Gamma$ crosses the Cartesian grid as is shown in Fig. 15. As the flow equations are solved on a staggered mesh, the squares in Fig. 15 denote horizontally oriented velocities. The body force $f_{i}$ is applied at velocity points in the fluid that have at least one direct neighbor in the solid. Balaras, ${ }^{21}$ working with the pressure-correction algorithm as well, proposes to split up the prediction step into one step without the body force $f_{i}$ and one step with the body force $f_{i}$. In this way, the predicted velocities exactly 


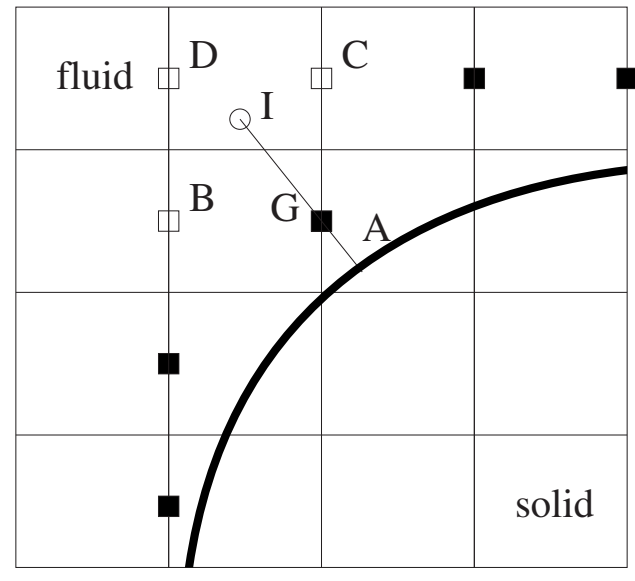

FIG. 15. A Cartesian grid with an immersed boundary. The filled squares denote boundary velocity nodes, where the body force $f_{i}$ is applied; the open squares denote standard velocity nodes.

satisfy the boundary conditions at the embedded boundary $\Gamma$. The imposition of the body force $f_{i}$, however, requires the reconstruction of the local velocity field, as $f_{i}$ is directly dependent on $V_{i}^{n+1}$. This velocity $V_{i}^{n+1}$ is obtained by means of interpolation.

\section{Reconstruction of the velocity field}

Reconstruction of the local velocity field by means of interpolation is needed at, for instance, point $G$ in Fig. 15. Many reconstruction methods in literature (for instance, Refs. 28-30) rely on linear, bilinear, or quadratic interpolation routines. However, if the first grid point near the wall is located outside the viscous sublayer, these types of interpolations are inappropriate and inadequate. As far as the authors know, only Tessicini et al. ${ }^{31}$ paid attention to this issue, when simulating a flow past a hydrofoil trailing edge. In this section, we describe how a proper velocity reconstruction, based on a certain wall function, can be applied.

Keeping Fig. 15 in mind, the algorithm can be described as follows.

(1) Locate all the fluid points that have at least one direct neighbor in the solid. An example is point $G$ in Fig. 15. These points will henceforth be referred to as boundary points.

(2) Find appropriate surrounding fluid points that are not boundary points at the same time. These points are denoted $B, C$, and $D$ in Fig. 15. Also determine the total velocity vectors at these points, which is necessary due to the staggered character of the grid.

(3) Find point $A$, which is defined as the point on the embedded boundary $\Gamma$, such that the distance $A G$ is minimal, i.e., the line $A G$ is perpendicular to the wall.

(4) Rotate the reference system locally, such that the tangent line at the embedded boundary $\Gamma$ and the line $A G$ become the new principal axes of the local reference system. The new axes are now denoted as $x_{t}$, the tangent direction, and $x_{n}$, the normal direction.

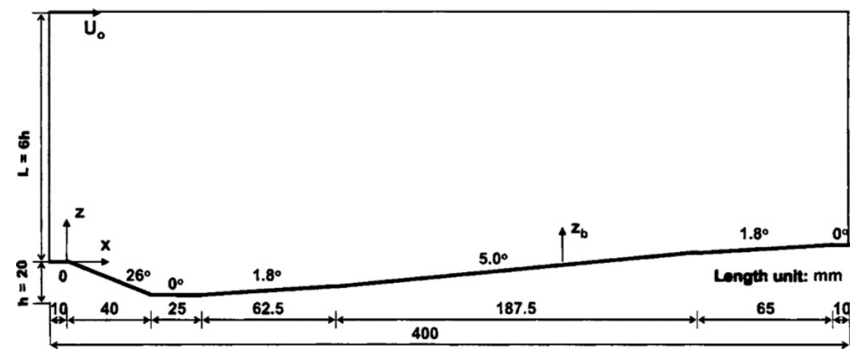

FIG. 16. The geometry of the fixed dune experiment of Yue et al. The amplitude of the bottom is denoted by $h$. The flow goes from left to right.

(5) Define an image point $I$ with coordinates $x_{t}^{I}=x_{t}^{G}$ and $x_{n}^{I}=\frac{1}{3}\left(x_{n}^{B}+x_{n}^{C}+x_{n}^{D}\right)$.

(6) Find the velocity components in tangent and normal direction in point $I$ by means of linear interpolation based on the polynomial $u=a_{1}+a_{2} x+a_{3} y$. At this point, linear interpolation is not inadequate since all the points $I, B$, $C$, and $D$ are located relatively far from the wall and relatively close to each other.

(7) Use the interpolated tangent velocity at point $I$ and the zero-velocity at point $A$ as boundary conditions for the determination of $u_{\tau}$ from Eq. (1) or Eq. (2). Use a linear velocity profile for the normal velocity from zero at point $A$ to the interpolated velocity at point $I$.

(8) Determine, from the solution from the previous step, the velocity components at point $G$.

(9) Translate the calculated velocity vector backward to the original reference system and use the velocity at point $G$ for the calculation of the body force $f_{i}$ in Eq. (A2). Notice here that due to the staggered character of the grid, only the horizontal component of the velocity at point $G$ is needed for the calculation of the body force $f_{i}$.

The above algorithm can easily be extended to threedimensional simulations. We now have formulated an easy extension of the method proposed by Balaras ${ }^{21}$ by the addition of the wall-model approach.

\section{Example: Open-channel flow over a fixed dune}

As an example, the open-channel flow over a fixed twodimensional dune studied by Yue et al. $^{32}$ is chosen (see Fig. 16). The geometry has a length of 20 times the dune height $h$ and a total channel depth of seven times the dune height. In Fig. 16, the flow goes from left to right. The Reynolds number is 57000 based on the channel height above the dune $(6 h)$ and the velocity at the free surface at the entry of the domain. Periodic boundary conditions are imposed in both the horizontal directions. The bottom is hydraulically smooth.

Two different grids are chosen to check the independency of the numerical solution of the chosen grid. The first grid, denoted as "fine," contains $288 \times 144 \times 224$ grid cells in streamwise, transverse, and vertical directions, respectively. The second grid, denoted as "coarse," contains 224 $\times 112 \times 176$ grid cells. This means that 32 and 25 grid cells, respectively, are located vertically along the dune height. On 

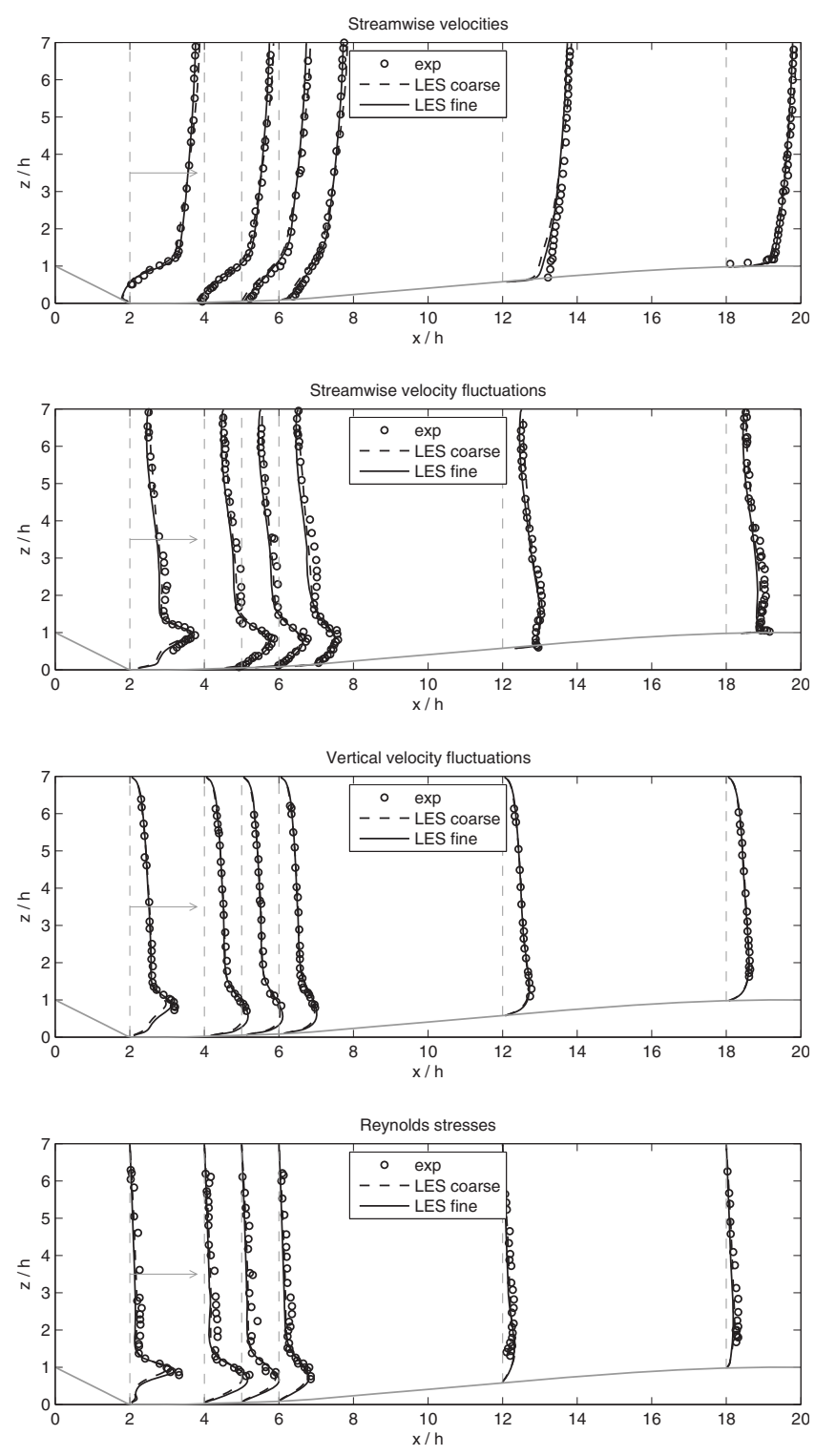

FIG. 17. From top to bottom: vertical profiles of the streamwise velocities, the streamwise velocity fluctuations, the vertical velocity fluctuations, and the Reynolds stresses $-\overline{v^{\prime} w^{\prime}}$ for the LES on two different grids. The open circles denote the experimental results taken from Ref. 32 . The quantities are scaled by means of the free surface velocity at the dune crest $U$. The arrows denote a magnitude of $0.1,0.2,0.2$, and 0.02 , respectively.

the upward sloping part, the distance between the first grid point from the wall and the wall itself is typically between 0 and 20 wall units.

Results for the streamwise velocities, the root-meansquare streamwise, and vertical velocities and the Reynolds stresses $-\overline{v^{\prime} w^{\prime}}$ are shown in Fig. 17 for the numerical results on the two grids as well as for the experimental results Ref. 32. The values are scaled by means of the free surface velocity at the dune crest $U$. It is seen from the vertical profiles of the streamwise velocities that the recirculation zone in the wake of the dune is very well captured. Mutual differences between the results obtained with the two grids can hardly be seen, indicating mesh independency. The numerical results for the second order statistics show a very good agreement with their experimental counterparts as well.
${ }^{1}$ I. L. Rozovskii, Flow of Water in Bends of Open Channels (Academy of Sciences of the Ukrainian SSR, Kiev, 1957; Israel Program for Scientific Translations, Jerusalem, 1961).

${ }^{2}$ A. J. Odgaard and M. A. Bergs, "Flow processes in a curved alluvial channel," Water Resour. Res. 24, 45, doi:10.1029/WR024i001p00045 (1988).

${ }^{3} \mathrm{~K}$. Blanckaert and W. H. Graf, "Experiments on flow in an open-channel bend: Mean flow and turbulence," J. Hydraul. Eng. 127, 835 (2001).

${ }^{4} \mathrm{R}$. Booij, "Measurements and large eddy simulations of the flows in some curved flumes," J. Turbul. 4, 8 (2003).

${ }^{5}$ J. D. Abad and M. H. Garcia, "Experiments in a high-amplitude Kinoshita meandering channel: 1. Implications of bend orientation on mean and turbulent flow structure," Water Resour. Res. 45, W02401, doi:10.1029/ 2008WR007016 (2009).

${ }^{6}$ J. D. Abad and M. H. Garcia, "Experiments in a high-amplitude Kinoshita meandering channel: 2. Implications of bend orientation on bed morphodynamics," Water Resour. Res. 45, W02402, doi:10.1029/ 2008WR007017 (2009).

${ }^{7}$ R. J. Calhoun and R. L. Street, "Turbulent flow over a wavy surface: Neutral case,” J. Geophys. Res., [Oceans] 106, 9277, doi:10.1029/ 2000JC900133 (2001).

${ }^{8}$ R. Broglia, A. Pascarelli, and U. Piomelli, "Large-eddy simulations of ducts with a free surface,” J. Fluid Mech. 484, 223 (2003).

${ }^{9}$ M. Koken and G. Constantinescu, "An investigation of the flow and scour mechanisms around isolated spur dikes in a shallow open channel: 2 . Conditions corresponding to the final stages of the erosion and deposition process," Water Resour. Res. 44, W08407, doi:10.1029/2007WR006491 (2008).

${ }^{10}$ T. Stoesser, N. Ruether, and N. R. B. Olsen, "Near-bed flow behavior in a meandering channel," Proceedings Riverflow 2008, Kubaba, Turkey, 2008, pp. 793-799.

${ }^{11}$ W. van Balen, W. S. J. Uijttewaal, and K. Blanckaert, "Large-eddy simulation of a mildly curved open-channel flow," J. Fluid Mech. 630, 413 (2009).

${ }^{12}$ W. van Balen, K. Blanckaert, and W. S. J. Uijttewaal, "Analysis of the role of turbulence in curved open-channel flow at different water depths by means of experiments, LES and RANS," J. Turbul. 11, 12 (2010).

${ }^{13} \mathrm{~K}$. Blanckaert, "Topographic steering, flow circulation, velocity redistribution and bed topography in sharp meander bends," Water Resour. Res., doi:10.1029/2009WR008303 (in press).

${ }^{14}$ J. Zeng, G. Constantinescu, K. Blanckaert, and L. Weber, "Flow and bathymetry in sharp open-channel bends: Experiments and predictions," Water Resour. Res. 44, W09401, doi:10.1029/2007WR006303 (2008).

${ }^{15}$ M. J. B. M. Pourquié, "Large-eddy simulation of a turbulent jet," Ph.D. thesis, Delft University of Technology, 1994.

${ }^{16}$ J. G. M. Eggels, F. Unger, M. H. Weiss, J. Westerweel, R. J. Adrian, R. Friedrich, and F. T. M. Nieuwstadt, "Fully developed turbulent pipe flow: A comparison between direct numerical simulation and experiment," J. Fluid Mech. 268, 175 (1994).

${ }^{17}$ Y. H. Tseng and J. H. Ferziger, "Large-eddy simulation of turbulent wavy boundary flow-illustration of vortex dynamics," J. Turbul. 5, 34 (2004).

${ }^{18}$ A. O. Demuren and W. Rodi, "Calculation of flow and pollutant dispersion in meandering channels," J. Fluid Mech. 172, 63 (1986).

${ }^{19}$ A. Vardy, Fluid Principles (McGraw-Hill, England, 1990).

${ }^{20}$ L. C. van Rijn, "Sediment transport, part 1: Bed load transport," J. Hydraul. Eng. 110, 1431 (1984).

${ }^{21} \mathrm{E}$. Balaras, "Modeling complex boundaries using an external force field on fixed Cartesian grids in large-eddy simulations," Comput. Fluids 33, 375 (2004).

${ }^{22}$ W. E. Dietrich and J. D. Smith, "Influence of the point bar on flow through curved channels," Water Resour. Res. 19, 1173, doi:10.1029/ WR019i005p01173 (1983).

${ }^{23}$ J. M. Nelson and J. D. Smith, "Flow in meandering channel with natural topography," in River Meandering, edited by S. Ikeda and G. Parker (AGU Water Resources Monograph, Washington, DC, 1989), pp. 69-102.

${ }^{24}$ S. B. Pope, Turbulent Flows (Cambridge University Press, Cambridge, England, 2000).

${ }^{25}$ J. L. Lumley, "Computational modeling of turbulent flows," Adv. Appl. Mech. 18, 123 (1979).

${ }^{26}$ A. J. Simonsen and P.-Å. Krogstad, "Turbulent stress invariant analysis: Clarification of existing terminology," Phys. Fluids 17, 088103 (2005). 
${ }^{27}$ K. Liu and R. H. Pletcher, "Anisotropy of a turbulent boundary layer," J. Turbul. 9, 18 (2008).

${ }^{28}$ E. A. Fadlun, R. Verzicco, P. Orlandi, and J. Mohd-Yusof, "Combined immersed-boundary finite-difference methods for three-dimensional complex flow simulations," J. Comput. Phys. 161, 35 (2000).

${ }^{29}$ J. Kim, D. Kim, and H. Choi, "An immersed boundary finite volume method for simulations of flow in complex geometries," J. Comput. Phys. 171, 132 (2001)
${ }^{30}$ Y.-H. Tseng and J. H. Ferziger, "A ghost-cell immersed boundary method for flow in complex geometry," J. Comput. Phys. 192, 593 (2003).

${ }^{31}$ F. Tessicini, G. Iaccarino, M. Fatica, M. Wang, and R. Verzicco, "Wall modeling for large-eddy simulation using an immersed boundary method," CTR Annual Research Briefs, 2002, pp. 181-187.

${ }^{32} \mathrm{~W}$. Yue, C. L. Lin, and V. C. Patel, "Large-eddy simulation of turbulent flow over a fixed two-dimensional dune," J. Hydraul. Eng. 132, 643 (2006). 